\title{
Orbital angular momentum at small $x$
}

\section{Yuri V. Kovchegov}

Department of Physics, The Ohio State University, 191 West Woodruff Avenue, Columbus, OH 43210, U.S.A.

E-mail: kovchegov.1@osu.edu

ABSTRACT: We determine the small Bjorken $x$ asymptotics of the quark and gluon orbital angular momentum (OAM) distributions in the proton in the double-logarithmic approximation (DLA), which resums powers of $\alpha_{s} \ln ^{2}(1 / x)$ with $\alpha_{s}$ the strong coupling constant. Starting with the operator definitions for the quark and gluon OAM, we simplify them at small $x$, relating them, respectively, to the polarized dipole amplitudes for the quark and gluon helicities defined in our earlier works. Using the small- $x$ evolution equations derived for these polarized dipole amplitudes earlier we arrive at the following small- $x$ asymptotics of the quark and gluon OAM distributions in the large- $N_{c}$ limit:

$$
\begin{gathered}
L_{q+\bar{q}}\left(x, Q^{2}\right)=-\Delta \Sigma\left(x, Q^{2}\right) \sim\left(\frac{1}{x}\right)^{\frac{4}{\sqrt{3}} \sqrt{\frac{\alpha_{s} N_{c}}{2 \pi}}}, \\
L_{G}\left(x, Q^{2}\right) \sim \Delta G\left(x, Q^{2}\right) \sim\left(\frac{1}{x}\right)^{\frac{13}{4 \sqrt{3}} \sqrt{\frac{\alpha_{s} N_{c}}{2 \pi}}} .
\end{gathered}
$$

KeYwords: Perturbative QCD, Resummation

ArXiv EPRINT: 1901.07453 


\section{Contents}

1 Introduction 1

2 Quark OAM 3

2.1 The quark OAM operator 3

2.2 Evaluation of the quark OAM operator at small $x \quad 4$

2.3 Evolution equations for quark OAM and their solution 9

2.4 Quark OAM distribution at small $x \quad 11$

3 Gluon OAM 12

3.1 The gluon OAM operator 12

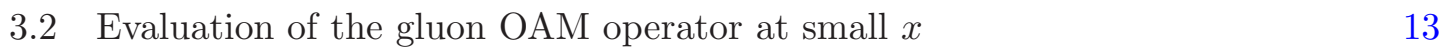

$\begin{array}{lll}3.3 & \text { Evolution equations for gluon OAM and their solution } & 18\end{array}$

$\begin{array}{ll}3.4 & \text { Gluon OAM distribution at small } x\end{array}$

$\begin{array}{llr}4 & \text { Summary } & 26\end{array}$

$\begin{array}{ll}\text { A The saturation/CGC averaging } & 27\end{array}$

$\begin{array}{ll}\text { B Solution of a useful system of integral equations } & 28\end{array}$

$\begin{array}{ll}\text { C Comparison with the earlier works } & 30\end{array}$

$\begin{array}{ll}\text { D Large nucleus limit } & 31\end{array}$

\section{Introduction}

In recent years a lot of progress has been achieved in our theoretical understanding of quark and gluon helicity distributions at small $x[1-6]$. While having precise control of helicity distributions is important for our understanding of the proton spin, another important component of the proton spin comes from the quark and gluon orbital angular momentum (OAM). The helicity sum rules [7-9] (see [10] for a review) include the quark and gluon contributions to the proton spin along with their OAM. Specifically, the Jaffe-Manohar sum rule [7] reads

$$
S_{q+\bar{q}}+L_{q+\bar{q}}+S_{G}+L_{G}=\frac{1}{2}
$$

Here

$$
S_{q+\bar{q}}\left(Q^{2}\right)=\frac{1}{2} \int_{0}^{1} d x \Delta \Sigma\left(x, Q^{2}\right) \quad \text { and } \quad S_{G}\left(Q^{2}\right)=\int_{0}^{1} d x \Delta G\left(x, Q^{2}\right),
$$


are the quark and gluon components of the proton spin expressed in terms of the quark and gluon helicity distributions $\Delta \Sigma\left(x, Q^{2}\right)=\sum_{f}\left[\Delta q^{f}\left(x, Q^{2}\right)+\Delta \bar{q}^{f}\left(x, Q^{2}\right)\right]$ and $\Delta G\left(x, Q^{2}\right)$. Importantly, $L_{q+\bar{q}}$ and $L_{G}$ in eq. (1.1) denote the quark and gluon OAM, respectively. Our understanding of the proton spin would be incomplete without a good quantitative understanding of $L_{q+\bar{q}}$ and $L_{G}$.

The quark and gluon OAM can be written down as integrals of their distributions in $x[11-15]$

$$
L_{q+\bar{q}}\left(Q^{2}\right)=\int_{0}^{1} d x L_{q+\bar{q}}\left(x, Q^{2}\right) \quad \text { and } \quad L_{G}\left(Q^{2}\right)=\int_{0}^{1} d x L_{G}\left(x, Q^{2}\right)
$$

It now becomes apparent that, just like the quark and gluon helicities, both the quark OAM and the gluon OAM may receive contributions from the small- $x$ region. Even if the difficulty in experimentally measuring the gluon OAM is somehow surmounted, any given experiment can measure $L_{q+\bar{q}}\left(x, Q^{2}\right)$ and $L_{G}\left(x, Q^{2}\right)$ only down to some minimal value of $x=x_{\text {min }}$. No matter how small are the values of $x_{\min }$ to be accessed in the future experiments, one always faces the question of constraining the $x<x_{\min }$ region. It appears that a solid theoretical understanding of $L_{q+\bar{q}}\left(x, Q^{2}\right)$ and $L_{G}\left(x, Q^{2}\right)$ at small $x$ is necessary to accomplish this goal. The aim of this paper is to theoretically determine the small- $x$ asymptotics of the quark and gluon OAM.

Important first steps in this direction were taken in [16], where the one-loop $Q^{2}$ evolution equations for $L_{q+\bar{q}}\left(x, Q^{2}\right)$ and $L_{G}\left(x, Q^{2}\right)[12,17,18]$ were solved both numerically and analytically, with the aim of determining the small- $x$ asymptotics of these quantities. However, $Q^{2}$ evolution equations at the one-loop level resum powers of $\alpha_{s} \ln Q^{2} / Q_{0}^{2}$ with some initial momentum scale $Q_{0}$. At small $x$ these equations correctly reproduce the powers of $\alpha_{s} \ln Q^{2} / Q_{0}^{2} \ln (1 / x)$. That is, they give one the small- $x$ asymptotics only at large values of $Q^{2}$. For spin-independent observables, the true small- $x$ asymptotics is obtained by resumming powers of $\alpha_{s} \ln (1 / x)$ (without any ordering of the transverse momenta): this is done in the Balitsky-Fadin-Kuraev-Lipatov (BFKL) [19, 20], Balitsky-Kovchegov (BK) [21-24] and Jalilian-Marian-Iancu-McLerran-Weigert-Leonidov-Kovner (JIMWLK) [25-28] evolution equations. For many spin-dependent observables, the leading small- $x$ contribution comes from resumming the powers of $\alpha_{s} \ln ^{2}(1 / x)$ [29-35] (such parameter does not exist in the unpolarized evolution). This is the double-logarithmic approximation (DLA). In the case of helicity, the resummation of $\alpha_{s} \ln ^{2}(1 / x)$ was addressed in $[1-6,36,37]$. Our aim here is to resum powers of $\alpha_{s} \ln ^{2}(1 / x)$ for $L_{q+\bar{q}}\left(x, Q^{2}\right)$ and $L_{G}\left(x, Q^{2}\right)$, obtaining the leading small- $x$ asymptotics for these important quantities. To achieve this goal we will build upon our prior experience with the gluon helicity at small $x$ [1-6].

Below we will start by analyzing the quark OAM at small $x$ in section 2 . We will first define the quark OAM operator using the quark Wigner distribution [38] in section 2.1 following [39-41]. We then employ the technique from $[5,6]$ to simplify the quark OAM operator at small $x$ in section 2.2. This leads to a relationship between $L_{q+\bar{q}}\left(x, Q^{2}\right)$ and the fundamental polarized dipole amplitude [1], which is an expectation value of the polarized fundamental dipole operator defined in [6]. While the quark helicity distribution was related 
to the impact-parameter integrated fundamental polarized dipole amplitude [1], the quark OAM is related to the first impact parameter moment of this amplitude, as defined in eqs. (2.32). Using the evolution equations constructed for the fundamental polarized dipole amplitude in $[1,6]$, in section 2.3 we construct and solve the evolution equations for the first impact parameter moment of the amplitude. The consequences of this solution for quark OAM at small $x$ are summarized in section 2.4 , with the resulting small- $x$ asymptotics of quark OAM distribution given by eq. (2.50) (at large $N_{c}$ and in DLA).

The gluon OAM distribution is analyzed in section 3 following the same general strategy. Using the gluon Wigner distribution the gluon OAM operator is constructed in section 3.1. It is simplified at small $x$ in section 3.2. The gluon OAM distribution, just like the gluon helicity, is related to a different polarized gluon dipole operator defined in $[5,18]$. Similar to the quark OAM case, the gluon OAM distribution is related to the first impact parameter moment of the polarized gluon dipole amplitude (see eq. (3.28)). The evolution equation for this moment is derived and solved in section 3.3. Evolution equations for gluon helicity are a bit more involved than those for quark helicity [5]: the same applies to OAMs. Finally, the solution is employed in section 3.4 to derive the small- $x$ asymptotics (3.74) of the gluon OAM distribution (in the DLA limit and at large $N_{c}$ ).

The results of our analysis are concisely summarized in the equations (1) in the Abstract above as well as in section 4 .

\section{Quark OAM}

\subsection{The quark OAM operator}

We start with a generic (quark or gluon) OAM written in terms of the Wigner distribution function $W(p, b)$,

$$
L_{z}=\int \frac{d^{2} b_{\perp} d b^{-} d^{2} k_{\perp} d k^{+}}{(2 \pi)^{3}}(\underline{b} \times \underline{k})_{z} W(k, b) .
$$

Our notation for the light-cone components of the 4-vectors is $v^{ \pm}=\left(v^{0} \pm v^{3}\right) / \sqrt{2}$, while the transverse vectors are defined as $\underline{v}=\left(v^{1}, v^{2}\right)$. We also denote $b=\left(b^{-}, \underline{b}\right)$ and $k=\left(k^{+}, \underline{k}\right)$.

To construct the quark OAM operator using eq. (2.1) we need the quark Wigner function. We can extract the Wigner function from the unpolarized quark transversemomentum dependent (TMD) distribution (in a longitudinally polarized proton) [42]

$$
f_{1}^{q}\left(x, k_{T}^{2}\right)=\frac{1}{(2 \pi)^{3}} \int d^{2} r d r^{-} e^{i k \cdot r}\left\langle P S_{L}\left|\bar{\psi}(0) \mathcal{U}[0, r] \frac{\gamma^{+}}{2} \psi(r)\right| P S_{L}\right\rangle_{r^{+}=0},
$$

with $k^{+}=x P^{+}$and $\mathcal{U}[0, r]$ a Wilson line staple connecting points $r$ and 0 . At small- $x$ and in the $A^{-}=0$ gauge we write this TMD (in the case of the semi-inclusive deep inelastic scattering (SIDIS) future-pointing staple) as [6]

$$
\begin{aligned}
f_{1}^{q}\left(x, k_{T}^{2}\right) & =\frac{2 P^{+}}{(2 \pi)^{3}} \sum_{X} \int d^{2} \zeta d \zeta^{-} d^{2} \xi d \xi^{-} e^{i k \cdot(\zeta-\xi)}\left\langle\bar{\psi}_{\alpha}(\xi) V_{\underline{\xi}}\left[\xi^{-}, \infty\right] \mid X\right\rangle\left(\frac{1}{2} \gamma^{+}\right)_{\alpha \beta}\left\langle X \mid V_{\underline{\zeta}}\left[\infty, \zeta^{-}\right] \psi_{\beta}(\zeta)\right\rangle \\
& \equiv \frac{P^{+}}{(2 \pi)^{3}} \int d^{2}\left(\frac{1}{2} \zeta_{\perp}+\frac{1}{2} \xi_{\perp}\right) d\left(\frac{1}{2} \zeta^{-}+\frac{1}{2} \xi^{-}\right) W^{q, \text { SIDIS }}\left(k, \frac{1}{2} \zeta+\frac{1}{2} \xi\right) .
\end{aligned}
$$


Here the large angle brackets denote the averaging in the target (proton) wave function, as is done in the saturation/color glass condensate (CGC) physics [43-49]. The averaging is discussed in appendix A in more detail and is given by eq. (A.4) there. We also use the following notation for the fundamental Wilson lines on the $x^{-}$light cone,

$$
V_{\underline{x}}\left[b^{-}, a^{-}\right]=\mathcal{P} \exp \left[i g \int_{a^{-}}^{b^{-}} d x^{-} A^{+}\left(x^{+}=0, x^{-}, \underline{x}\right)\right] .
$$

The second line in eq. (2.3) is the definition of the quark SIDIS Wigner distribution, which we can use to obtain

$$
\begin{array}{r}
W^{q, \operatorname{SIDIS}}(k, b)=2 \sum_{X} \int d^{2} r d r^{-} e^{i k \cdot r}\left\langle\bar{\psi}_{\alpha}\left(b-\frac{1}{2} r\right) V_{\underline{b}-\frac{1}{2} \underline{\underline{r}}}\left[b^{-}-\frac{1}{2} r^{-}, \infty\right] \mid X\right\rangle\left(\frac{1}{2} \gamma^{+}\right)_{\alpha \beta} \\
\times\left\langle X \mid V_{\underline{b}+\frac{1}{2} \underline{r}}\left[\infty, b^{-}+\frac{1}{2} r^{-}\right] \psi_{\beta}\left(b+\frac{1}{2} r\right)\right\rangle .
\end{array}
$$

Inserting this into eq. (2.1) yields

$$
\begin{array}{r}
L_{q}\left(Q^{2}\right)=\frac{2 P^{+}}{(2 \pi)^{3}} \sum_{X} \int d^{2} k_{\perp} d x d^{2} \zeta d \zeta^{-} d^{2} \xi d \xi^{-} e^{i k \cdot(\zeta-\xi)}\left(\frac{\underline{\zeta}+\underline{\xi}}{2} \times \underline{k}\right)\left\langle\bar{\psi}_{\alpha}(\xi) V_{\underline{\xi}}\left[\xi^{-}, \infty\right] \mid X\right\rangle\left(\frac{1}{2} \gamma^{+}\right)_{\alpha \beta} \\
\times\left\langle X \mid V_{\underline{\zeta}}\left[\infty, \zeta^{-}\right] \psi_{\beta}(\zeta)\right\rangle .
\end{array}
$$

(Note that the $x$-integral from here on is assumed to run from 0 to 1.) We are particularly interested in the quark OAM distribution, $L_{q}\left(x, Q^{2}\right)=d L_{q}\left(Q^{2}\right) / d x$, which can be easily read from eq. (2.6),

$$
\begin{array}{r}
L_{q}\left(x, Q^{2}\right)=\frac{2 P^{+}}{(2 \pi)^{3}} \sum_{X} \int d^{2} k_{\perp} d^{2} \zeta d \zeta^{-} d^{2} \xi d \xi^{-} e^{i k \cdot(\zeta-\xi)}\left(\frac{\underline{\zeta}+\underline{\xi}}{2} \times \underline{k}\right)\left\langle\bar{\psi}_{\alpha}(\xi) V_{\underline{\xi}}\left[\xi^{-}, \infty\right] \mid X\right\rangle\left(\frac{1}{2} \gamma^{+}\right)_{\alpha \beta} \\
\times\left\langle X \mid V_{\underline{\zeta}}\left[\infty, \zeta^{-}\right] \psi_{\beta}(\zeta)\right\rangle .
\end{array}
$$

\subsection{Evaluation of the quark OAM operator at small $x$}

We now need to simplify the expression (2.7) for the quark OAM distribution at small $x$. Following the technique described in [6] (see eq. (10) there) we write

$$
\begin{aligned}
L_{q}\left(x, Q^{2}\right)=\frac{2 P^{+}}{(2 \pi)^{3}} \sum_{\bar{q}} \int d^{2} k_{\perp} d^{2} \zeta d^{2} \xi & \int_{-\infty}^{0} d \zeta^{-} \int_{0}^{\infty} d \xi^{-} e^{i k \cdot(\zeta-\xi)}\left(\frac{\underline{\zeta}+\underline{\xi}}{2} \times \underline{k}\right)\left(\frac{1}{2} \gamma^{+}\right)_{\alpha \beta} \\
& \times\left\langle\bar{\psi}_{\alpha}(\xi) \mid \bar{q}\right\rangle\left\langle\bar{q} \mid V_{\underline{\zeta}}[\infty,-\infty] \psi_{\beta}(\zeta)\right\rangle+\text { c.c. }
\end{aligned}
$$


For $\zeta^{-}<0$ and $\xi^{-}>0$ one can write down the quark propagator through the shock wave as $[5,6]$

$$
\begin{gathered}
\bar{\psi}_{\alpha}^{i}(\xi) \psi_{\beta}^{j}(\zeta)=\int d^{2} w \frac{d^{2} k_{1} d k_{1}^{-}}{(2 \pi)^{3}} \frac{d^{2} k_{2}}{(2 \pi)^{2}} e^{i \frac{\underline{k}_{1}^{2}}{2 k_{1}^{-}} \zeta^{-}-i \frac{\underline{k}_{2}^{2}}{2 k_{1}^{-}} \xi^{-}+i \underline{k}_{1} \cdot(\underline{w}-\underline{\zeta})+i \underline{k}_{2} \cdot(\underline{\xi}-\underline{w})} \theta\left(k_{1}^{-}\right) \\
\times\left.\left\{\left[\frac{k / 1}{2 k_{1}^{-}}\right]\left[\left(\hat{V}_{\underline{w}}^{\dagger}\right)^{j i}\right]\left[\frac{k_{2}}{2 k_{1}^{-}}\right]\right\}_{\beta \alpha}\right|_{k_{2}^{-}=k_{1}^{-}, k_{1}^{2}=0, k_{2}^{2}=0}
\end{gathered}
$$

such that

$$
\begin{aligned}
L_{q}\left(x, Q^{2}\right)=\frac{2 P^{+}}{(2 \pi)^{3}} & \int d^{2} k_{\perp} d^{2} \zeta d^{2} \xi \int_{-\infty}^{0} d \zeta^{-} \int_{0}^{\infty} d \xi^{-} e^{i k \cdot(\zeta-\xi)}\left(\frac{\underline{\zeta}+\underline{\xi}}{2} \times \underline{k}\right) \\
& \times \int d^{2} w \frac{d^{2} k_{1} d k_{1}^{-}}{(2 \pi)^{3}} \frac{d^{2} k_{2}}{(2 \pi)^{2}} e^{\left.i \frac{\underline{k}_{1}^{2}}{2 k_{1}^{-}} \zeta^{-}-i \frac{\underline{k}_{2}^{2}}{2 k_{1}^{-}} \xi^{-}+i \underline{k}_{1} \cdot \underline{w}-\underline{\zeta}\right)+i \underline{k}_{2} \cdot \underline{\underline{\xi}-\underline{w})}} \theta\left(k_{1}^{-}\right)\left(\frac{1}{2} \gamma^{+}\right)_{\alpha \beta} \\
& \times\left\langle\left.\mathrm{T} V_{\underline{\underline{\zeta}}}^{i j}[\infty,-\infty]\left\{\left[\frac{k / 1}{2 k_{1}^{-}}\right]\left[\left(\hat{V}_{\underline{w}}^{\dagger}\right)^{j i}\right]\left[\frac{k / 2}{2 k_{1}^{-}}\right]\right\}_{\beta \alpha}\right|_{k_{2}^{-}=k_{1}^{-}, k_{1}^{2}=0, k_{2}^{2}=0}\right\rangle+\text { c.c. . }
\end{aligned}
$$

As in [6], we are using the time-ordering sign $\mathrm{T}$ to delineate the amplitude from the complex conjugate amplitude, with the latter containing the anti-time ordering sign $\overline{\mathrm{T}}$. Integrating over $\zeta^{-}$and $\xi^{-}$and neglecting higher powers of $x$ yields

$$
\begin{aligned}
& L_{q}\left(x, Q^{2}\right)=-\frac{2 P^{+}}{(2 \pi)^{3}} \int d^{2} k_{\perp} d^{2} \zeta d^{2} \xi e^{-i \underline{k} \cdot(\underline{\zeta}-\underline{\xi})}\left(\frac{\underline{\zeta}+\underline{\xi}}{2} \times \underline{k}\right) \\
& \times \int d^{2} w \frac{d^{2} k_{1} d k_{1}^{-}}{(2 \pi)^{3}} \frac{d^{2} k_{2}}{(2 \pi)^{2}} e^{i \underline{k}_{1} \cdot(\underline{w}-\underline{\zeta})+i \underline{k}_{2} \cdot(\underline{\xi}-\underline{w})} \theta\left(k_{1}^{-}\right)\left(\frac{1}{2} \gamma^{+}\right)_{\alpha \beta} \\
& \times\left\langle\left.\mathrm{T} V_{\underline{\zeta}}^{i j}[\infty,-\infty]\left\{\frac{k_{1}}{\underline{k}_{1}^{2}}\left(\hat{V}_{\underline{w}}^{\dagger}\right)^{j i} \frac{k_{2}}{\underline{k}_{2}^{2}}\right\}_{\beta \alpha}\right|_{k_{2}^{-}=k_{1}^{-}, k_{1}^{2}=0, k_{2}^{2}=0}\right\rangle+\text { c.c. . }
\end{aligned}
$$

Employing polarization sums we write

$$
\begin{aligned}
L_{q}\left(x, Q^{2}\right)= & -\frac{2 P^{+}}{(2 \pi)^{3}} \int d^{2} k_{\perp} d^{2} \zeta d^{2} \xi e^{-i \underline{k} \cdot(\underline{\zeta}-\underline{\xi})}\left(\frac{\underline{\zeta}+\underline{\xi}}{2} \times \underline{k}\right) \\
& \times \int d^{2} w \frac{d^{2} k_{1} d k_{1}^{-}}{(2 \pi)^{3}} \frac{d^{2} k_{2}}{(2 \pi)^{2}} e^{i \underline{k}_{1} \cdot(\underline{w}-\underline{\zeta})+i \underline{k}_{2} \cdot(\underline{\xi}-\underline{w})} \theta\left(k_{1}^{-}\right) \sum_{\sigma_{1}, \sigma_{2}} \bar{v}_{\sigma_{2}}\left(k_{2}\right) \frac{1}{2} \gamma^{+} v_{\sigma_{1}}\left(k_{1}\right) \\
& \times\left\langle\left.\mathrm{T} V_{\underline{\zeta}}^{i j}[\infty,-\infty] \frac{1}{\underline{k}_{1}^{2} \underline{k}_{2}^{2}} \bar{v}_{\sigma_{1}}\left(k_{1}\right)\left(\hat{V}_{\underline{w}}^{\dagger}\right)^{j i} v_{\sigma_{2}}\left(k_{2}\right)\right|_{k_{2}^{-}=k_{1}^{-}, k_{1}^{2}=0, k_{2}^{2}=0}\right\rangle+\text { c.c. }
\end{aligned}
$$


The polarized "Wilson line" is defined by [6]

$$
\left[\bar{v}_{\sigma}(p)\left(\hat{V}_{\underline{x}}^{\dagger}\right) v_{\sigma^{\prime}}\left(p^{\prime}\right)\right]=2 \sqrt{p^{-} p^{\prime}} \delta_{\sigma \sigma^{\prime}}\left(V_{\underline{x}}^{\dagger}-\sigma V_{\underline{x}}^{\mathrm{pol} \dagger}+\ldots\right)=2 \sqrt{p^{-} p^{\prime}} \delta_{\sigma \sigma^{\prime}} V_{\underline{x}}^{\dagger}(-\sigma)+\ldots,
$$

where ellipsis denote polarization-independent sub-eikonal terms, which are not important for our calculation. Here we employ an abbreviated notation $V_{\underline{x}} \equiv V_{\underline{x}}[\infty,-\infty]$. We will use the $+\leftrightarrow-$ interchanged Brodsky-Lepage (BL) spinors [50], which we will also refer to as the anti-BL spinors $[6,51]$ :

$u_{\sigma}(p)=\frac{1}{\sqrt{\sqrt{2} p^{-}}}\left[\sqrt{2} p^{-}+m \gamma^{0}+\gamma^{0} \underline{\gamma} \cdot \underline{p}\right] \rho(\sigma), \quad v_{\sigma}(p)=\frac{1}{\sqrt{\sqrt{2} p^{-}}}\left[\sqrt{2} p^{-}-m \gamma^{0}+\gamma^{0} \underline{\gamma} \cdot \underline{p}\right] \rho(-\sigma)$,

where $p^{\mu}=\left(\frac{\underline{p}^{2}+m^{2}}{2 p^{-}}, p^{-}, \underline{p}\right)$ and

$$
\rho(+1)=\frac{1}{\sqrt{2}}\left(\begin{array}{c}
1 \\
0 \\
-1 \\
0
\end{array}\right), \quad \rho(-1)=\frac{1}{\sqrt{2}}\left(\begin{array}{l}
0 \\
1 \\
0 \\
1
\end{array}\right) .
$$

Using these spinors with massless quarks we get

$$
\bar{v}_{\sigma_{2}}\left(k_{2}\right) \frac{1}{2} \gamma^{+} v_{\sigma_{1}}\left(k_{1}\right)=\frac{1}{2} \delta_{\sigma_{2} \sigma_{1}} \frac{\left(\underline{k}_{2} \cdot \underline{k}_{1}\right)+i \sigma_{1}\left(\underline{k}_{1} \times \underline{k}_{2}\right)}{\sqrt{k_{1}^{-} k_{2}^{-}}} .
$$

Performing the sum over $\sigma_{1}, \sigma_{2}$ in eq. (2.12) with the help of eqs. (2.13) and (2.16) we arrive at

$$
\begin{aligned}
& L_{q}\left(x, Q^{2}\right)=-\frac{4 P^{+}}{(2 \pi)^{3}} \int d^{2} k_{\perp} d^{2} \zeta d^{2} \xi e^{-i \underline{k} \cdot(\underline{\zeta}-\underline{\xi})}\left(\frac{\underline{\zeta}+\underline{\xi}}{2} \times \underline{k}\right) \\
& \times \int d^{2} w \frac{d^{2} k_{1} d k_{1}^{-}}{(2 \pi)^{3}} \frac{d^{2} k_{2}}{(2 \pi)^{2}} e^{i \underline{k}_{1} \cdot(\underline{w}-\underline{\zeta})+i \underline{k_{2}} \cdot(\underline{\xi}-\underline{w})} \theta\left(k_{1}^{-}\right) \frac{1}{\underline{k}_{1}^{2} \underline{k}_{2}^{2}} \\
& \times\left\langle\underline{k}_{1} \cdot \underline{k}_{2} \operatorname{Ttr}\left[V_{\underline{\zeta}} V_{\underline{w}}^{\dagger}\right]-i \underline{k}_{1} \times \underline{k}_{2} \operatorname{Ttr}\left[V_{\underline{\zeta}} V_{\underline{w}}^{\mathrm{pol} \dagger}\right]\right\rangle+\text { c.c. } .
\end{aligned}
$$

Next we integrate over $\underline{k}_{1}$ and $\underline{k}_{2}$. This yields

$$
\begin{aligned}
& L_{q}\left(x, Q^{2}\right)=-\frac{4 P^{+}}{(2 \pi)^{5}} \int d^{2} k_{\perp} d^{2} \zeta d^{2} \xi d^{2} w e^{-i \underline{k} \cdot(\underline{\zeta}-\underline{\xi})}\left(\frac{\underline{\zeta}+\underline{\xi}}{2} \times \underline{k}\right) \int_{0}^{\infty} \frac{d k_{1}^{-}}{2 \pi} \\
& \times\left\{\frac{\underline{\zeta}-\underline{w}}{|\underline{\zeta}-\underline{w}|^{2}} \cdot \frac{\underline{\xi}-\underline{w}}{|\underline{\xi}-\underline{w}|^{2}}\left\langle\mathrm{~T} \operatorname{tr}\left[V_{\zeta} V_{\underline{w}}^{\dagger}\right]\right\rangle-i \frac{\underline{\zeta}-\underline{w}}{|\underline{\zeta}-\underline{w}|^{2}} \times \frac{\underline{\xi}-\underline{w}}{|\underline{\xi}-\underline{w}|^{2}}\left\langle\mathrm{~T} \operatorname{tr}\left[V_{\underline{\zeta}} V_{\underline{w}}^{\mathrm{pol} \dagger}\right]\right\rangle\right\}+\text { c.c. . }
\end{aligned}
$$

Adding the complex conjugate we obtain

$$
\begin{aligned}
& L_{q}\left(x, Q^{2}\right)=-\frac{4 P^{+}}{(2 \pi)^{5}} \int d^{2} k_{\perp} d^{2} \zeta d^{2} \xi d^{2} w e^{-i \underline{k} \cdot(\underline{\underline{\zeta}}-\underline{\underline{\xi}})}\left(\frac{\underline{\zeta}+\underline{\xi}}{2} \times \underline{k}\right) \int_{0}^{\infty} \frac{d k_{1}^{-}}{2 \pi}
\end{aligned}
$$

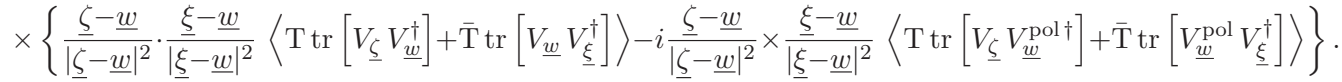


In the second term of each angle brackets we replace $\underline{k} \rightarrow-\underline{k}$ and interchange $\underline{\zeta} \leftrightarrow \underline{\xi}$ :

$$
\begin{aligned}
L_{q}\left(x, Q^{2}\right)= & -\frac{4 P^{+}}{(2 \pi)^{5}} \int d^{2} k_{\perp} d^{2} \zeta d^{2} \xi d^{2} w e^{-i \underline{k} \cdot(\underline{\zeta}-\underline{\xi})}\left(\frac{\underline{\zeta}+\underline{\xi}}{2} \times \underline{k}\right) \int_{0}^{\infty} \frac{d k_{1}^{-}}{2 \pi} \\
& \times\left\{\frac{\underline{\zeta}-\underline{w}}{|\underline{\zeta}-\underline{w}|^{2}} \cdot \frac{\underline{\xi}-\underline{w}}{|\underline{\xi}-\underline{w}|^{2}}\left\langle\operatorname{T} \operatorname{tr}\left[V_{\underline{\zeta}} V_{\underline{w}}^{\dagger}\right]-\overline{\mathrm{T}} \operatorname{tr}\left[V_{\underline{w}} V_{\underline{\underline{c}}}^{\dagger}\right]\right\rangle-i \frac{\underline{\zeta}-\underline{w}}{|\underline{\zeta}-\underline{w}|^{2}} \times \frac{\underline{\xi}-\underline{w}}{|\underline{\xi}-\underline{w}|^{2}}\left\langle\operatorname{T} \operatorname{tr}\left[V_{\underline{\underline{\zeta}}} V_{\underline{w}}^{\mathrm{pol} \dagger}\right]+\overline{\mathrm{T}} \operatorname{tr}\left[V_{\underline{w}}^{\mathrm{pol}} V_{\underline{\zeta}}^{\dagger}\right]\right\rangle\right\} .
\end{aligned}
$$

Employing the reflection symmetry with respect to the final-state cut, or, equivalently using eqs. (22) from [6] we conclude that (cf. eq. (24) in [6])

$$
\left\langle\operatorname{T} \operatorname{tr}\left[V_{\underline{\zeta}} V_{\underline{w}}^{\dagger}\right]-\overline{\mathrm{T}} \operatorname{tr}\left[V_{\underline{w}} V_{\underline{\zeta}}^{\dagger}\right]\right\rangle=\left\langle\operatorname{tr}\left[V_{\underline{\zeta}} V_{\underline{w}}^{\dagger}\right]-\operatorname{tr}\left[V_{\underline{\zeta}}^{\dagger} V_{\underline{w}}\right]\right\rangle=0,
$$

where the last step employed the same reflection symmetry, which has been verified up to NLO in the unpolarized small- $x$ evolution [52]. We are thus left with

$$
\begin{aligned}
L_{q}\left(x, Q^{2}\right)= & \frac{4 P^{+} i}{(2 \pi)^{5}} \int d^{2} k_{\perp} d^{2} \zeta d^{2} \xi d^{2} w e^{-i \underline{k} \cdot(\underline{\zeta}-\underline{\xi})}\left(\frac{\underline{\zeta}+\underline{\xi}}{2} \times \underline{k}\right) \int_{0}^{\infty} \frac{d k_{1}^{-}}{2 \pi} \\
& \times\left[\frac{\underline{\zeta}-\underline{w}}{|\underline{\zeta}-\underline{w}|^{2}} \times \frac{\underline{\xi}-\underline{w}}{|\underline{\xi}-\underline{w}|^{2}}\right]\left\langle\mathrm{T} \operatorname{tr}\left[V_{\underline{\zeta}} V_{\underline{w}}^{\mathrm{pol} \dagger}\right]+\overline{\mathrm{T}} \operatorname{tr}\left[V_{\underline{w}}^{\mathrm{pol}} V_{\underline{\zeta}}^{\dagger}\right]\right\rangle .
\end{aligned}
$$

For the flavor-singlet case we need to add the antiquark contribution. This yields

$$
\begin{aligned}
L_{q+\bar{q}}\left(x, Q^{2}\right)= & \frac{4 P^{+} i}{(2 \pi)^{5}} \int d^{2} k_{\perp} d^{2} \zeta d^{2} \xi d^{2} w e^{-i \underline{k} \cdot(\underline{\zeta}-\underline{\xi})}\left(\frac{\underline{\zeta}+\underline{\xi}}{2} \times \underline{k}\right) \int_{0}^{\infty} \frac{d k_{1}^{-}}{2 \pi} \\
& \times\left[\frac{\underline{\zeta}-\underline{w}}{|\underline{\zeta}-\underline{w}|^{2}} \times \frac{\underline{\xi}-\underline{w}}{|\underline{\xi}-\underline{w}|^{2}}\right]\left\langle\mathrm{T} \operatorname{tr}\left[V_{\underline{\zeta}} V_{\underline{w}}^{\mathrm{pol} \dagger}\right]+\mathrm{T} \operatorname{tr}\left[V_{\underline{w}}^{\mathrm{pol}} V_{\underline{\zeta}}^{\dagger}\right]+\overline{\mathrm{T}} \operatorname{tr}\left[V_{\underline{w}}^{\mathrm{pol}} V_{\underline{\zeta}}^{\dagger}\right]+\overline{\mathrm{T}} \operatorname{tr}\left[V_{\underline{\zeta}} V_{\underline{w}}^{\mathrm{pol} \dagger}\right]\right\rangle .
\end{aligned}
$$

Using the definition of the polarized dipole amplitude [6]

$$
G_{\underline{w}, \underline{\zeta}}(z s)=\frac{k_{1}^{-} P^{+}}{N_{c}} \operatorname{Re}\left\langle\operatorname{T} \operatorname{tr}\left[V_{\underline{\zeta}} V_{\underline{w}}^{\dagger \text { pol }}\right]+\mathrm{T} \operatorname{tr}\left[V_{\underline{w}}^{\text {pol }} V_{\underline{\zeta}}^{\dagger}\right]\right\rangle
$$

with $z s=2 k_{1}^{-} P^{+}$and inserting proper limits of the $k_{1}^{-}$integration we rewrite the contribution of eq. (2.23) as (see [6] for details)

$L_{q+\bar{q}}\left(x, Q^{2}\right)=\frac{8 i N_{c}}{(2 \pi)^{6}} \int d^{2} k_{\perp} d^{2} \zeta d^{2} \xi d^{2} w e^{-i \underline{k} \cdot(\underline{\zeta}-\underline{\xi})}\left(\frac{\underline{\zeta}+\underline{\xi}}{2} \times \underline{k}\right) \int_{\Lambda^{2} / s}^{Q^{2} /(x s) \approx 1} \frac{d z}{z} \frac{\underline{\zeta}-\underline{w}}{|\underline{\zeta}-\underline{w}|^{2}} \times \frac{\underline{\xi}-\underline{w}}{|\underline{\xi}-\underline{w}|^{2}} G_{\underline{w}, \underline{\zeta}}(z s)$.

Here $s \approx Q^{2} / x$ is the center-of-mass energy squared for the polarized dipole-target system, $z$ is the minus momentum fraction of the dipole momentum carried by the soft quark or anti-quark line, and $\Lambda$ is the infrared (IR) cutoff. 
The expression (2.25) can be integrated over $\underline{\xi}$ :

$L_{q+\bar{q}}\left(x, Q^{2}\right)=\frac{8 i N_{c}}{(2 \pi)^{6}} \int d^{2} k_{\perp} d^{2} \zeta d^{2} w e^{-i \underline{k} \cdot(\underline{\zeta}-\underline{w})} \int_{\Lambda^{2} / s}^{1} \frac{d z}{z}\left[2 \pi i \frac{\underline{\zeta}-\underline{w}}{|\underline{\zeta}-\underline{w}|^{2}} \times \frac{\underline{k}}{\underline{k}^{2}}\left(\frac{\underline{\zeta}+\underline{w}}{2} \times \underline{k}\right)-\pi \frac{\underline{\zeta}-\underline{w}}{|\underline{\zeta}-\underline{w}|^{2}} \cdot \frac{\underline{k}}{\underline{k}^{2}}\right] G_{\underline{w}, \underline{\zeta}}(z s)$.

If we replace $\underline{w} \rightarrow \underline{x}_{1}$ and $\underline{\zeta} \rightarrow \underline{x}_{0}$, and use the integration variables $\underline{x}_{10}=\underline{x}_{1}-\underline{x}_{0}$ and $\underline{x}_{1}$, then eq. (2.26) can be rewritten as

$L_{q+\bar{q}}\left(x, Q^{2}\right)=\frac{8 N_{c}}{(2 \pi)^{5}} \int d^{2} k_{\perp} d^{2} x_{10} d^{2} x_{1} e^{i \underline{k} \underline{\underline{k}} \underline{x}_{10}} \frac{\underline{x}_{10}}{x_{10}^{2}} \times \frac{\underline{k}}{\underline{k}^{2}} \underline{x}_{1} \times \underline{k} \int_{\Lambda^{2 / s}}^{1} \frac{d z}{z} G_{10}(z s)-\sum_{f}\left[\Delta q^{f}\left(x, Q^{2}\right)+\Delta \bar{q}^{f}\left(x, Q^{2}\right)\right]$,

where we have summed over flavors and, for each flavor,

$$
\Delta q^{f}\left(x, Q^{2}\right)+\Delta \bar{q}^{f}\left(x, Q^{2}\right)=\int d^{2} k_{\perp} g_{1 L}^{S}\left(x, k_{T}^{2}\right)
$$

with the flavor-singlet SIDIS quark helicity TMD at small $x[6]$

$$
g_{1 L}^{S}\left(x, k_{T}^{2}\right)=\frac{8 i N_{c}}{(2 \pi)^{5}} \int d^{2} \zeta d^{2} w e^{-i \underline{k} \cdot(\underline{\zeta}-\underline{w})} \int_{\Lambda^{2} / s}^{1} \frac{d z}{z} \frac{\underline{\zeta}-\underline{w}}{|\underline{\zeta}-\underline{w}|^{2}} \cdot \frac{\underline{k}}{\underline{k}^{2}} G_{\underline{w}, \underline{\zeta}}(z s) .
$$

In arriving at $(2.27)$ we have used the fact that, for fixed $\underline{x}_{10}$, the $x_{1}$-integral

$$
\int d^{2} x_{1} G_{10}(z s) \equiv G\left(x_{10}^{2}, z s\right)
$$

is a function of $x_{10}^{2}$ only. (In our notation $\underline{x}_{i j}=\underline{x}_{i}-\underline{x}_{j}$ and $x_{i j}=\left|\underline{x}_{i}-\underline{x}_{j}\right|$ for any $i, j$.)

At this point it may be tempting to conclude that since the small- $x$ /large- $z s$ asymptotics of quark helicity distribution $\Delta q^{f}\left(x, Q^{2}\right)$ and $G\left(x_{10}^{2}, z s\right)$ were derived in $[2,4]$, then the small- $x$ asymptotics of the quark OAM distribution $L_{q+\bar{q}}\left(x, Q^{2}\right)$ follows straightforwardly from eq. (2.27). This is almost correct, with one caveat: in $[2,4]$ we found the asymptotics of $G\left(x_{10}^{2}, z s\right)$, as defined in eq. (2.30), that is, of $G_{10}(z s)$ integrated over all impact parameters, since this is what $\Delta \Sigma\left(x, Q^{2}\right)$ depends on. In eq. (2.27), in the first term on its right-hand side, we need a different object: we need the "first moment" of $G_{10}(z s)$ in the impact parameter $\left(\underline{x}_{1}\right)$ space,

$$
\int d^{2} x_{1} x_{1}^{k} G_{10}(z s)
$$

Here the index $k=1,2$. Our next step is to determine the small- $x$ /large- $z s$ asymptotics of the "moment" in eq. (2.31). 


\subsection{Evolution equations for quark OAM and their solution}

Define

$$
\begin{aligned}
I^{k}\left(\underline{x}_{10}, z s\right) & =\int d^{2} x_{1} x_{1}^{k} G_{10}(z s), \\
J^{k}\left(\underline{x}_{10}, x_{21}^{2}, z s\right) & =\int d^{2} x_{1} x_{1}^{k} \Gamma_{10,21}(z s) .
\end{aligned}
$$

The evolution for these new objects in the large- $N_{c}$ DLA approximation can be found from eqs. (80) and (82) of [1] for the polarized dipole amplitude $G_{10}(z)$ and an auxiliary function, the polarized neighbor dipole amplitude $\Gamma_{10,21}\left(z^{\prime}\right)[1,6]$ (with the $S$-matrix for the unpolarized dipole amplitude taken to be $S=1$ in those equations):

$$
\begin{aligned}
G_{10}(z s)= & G_{10}^{(0)}(z s)+\frac{\alpha_{s} N_{c}}{2 \pi^{2}} \int_{\frac{1}{s x_{10}^{2}}}^{z} \frac{d z^{\prime}}{z^{\prime}} \int \frac{d^{2} x_{2}}{x_{21}^{2}} \theta\left(x_{10}-x_{21}\right) \theta\left(x_{21}^{2}-\frac{1}{z^{\prime} s}\right)\left[\Gamma_{10,21}\left(z^{\prime} s\right)+3 G_{21}\left(z^{\prime} s\right)\right], \\
\Gamma_{10,21}\left(z^{\prime} s\right)= & G_{10}^{(0)}\left(z^{\prime} s\right)+\frac{\alpha_{s} N_{c}}{2 \pi^{2}} \int_{\min \left\{\Lambda^{2}, \frac{1}{x_{10}^{2}}\right\} / s}^{z^{\prime}} \frac{d z^{\prime \prime}}{z^{\prime \prime}} \int \frac{d^{2} x_{3}}{x_{32}^{2}} \theta\left(\min \left\{x_{10}^{2}, x_{21}^{2} z^{\prime} / z^{\prime \prime}\right\}-x_{32}^{2}\right) \theta\left(x_{32}^{2}-\frac{1}{z^{\prime \prime} s}\right) \\
& \times\left[\Gamma_{10,32}\left(z^{\prime \prime} s\right)+3 G_{32}\left(z^{\prime \prime} s\right)\right] .
\end{aligned}
$$

Multiplying both sides by $x_{1}^{k}$ and integrating over $x_{1}$ while keeping $\underline{x}_{10}$ fixed we get

$$
\begin{gathered}
I^{k}\left(\underline{x}_{10}, z s\right)=I^{(0) k}\left(\underline{x}_{10}, z s\right)+\frac{\alpha_{s} N_{c}}{2 \pi^{2}} \int_{\frac{1}{s x_{10}^{2}}}^{z} \frac{d z^{\prime}}{z^{\prime}} \int \frac{d^{2} x_{21}}{x_{21}^{2}} \theta\left(x_{10}-x_{21}\right) \theta\left(x_{21}^{2}-\frac{1}{z^{\prime} s}\right) J^{k}\left(\underline{x}_{10}, x_{21}^{2}, z^{\prime} s\right), \\
J^{k}\left(\underline{x}_{10}, x_{21}^{2}, z^{\prime} s\right)=I^{(0) k}\left(\underline{x}_{10}, z^{\prime} s\right)+\frac{\alpha_{s} N_{c}}{2 \pi^{2}} \int_{\min \left\{\Lambda^{2}, \frac{1}{x_{10}^{2}}\right\} / s}^{z^{\prime}} \frac{d z^{\prime \prime}}{z^{\prime \prime}} \int \frac{d^{2} x_{32}}{x_{32}^{2}} \\
\\
\times \theta\left(\min \left\{x_{10}^{2}, x_{21}^{2} z^{\prime} / z^{\prime \prime}\right\}-x_{32}^{2}\right) \theta\left(x_{32}^{2}-\frac{1}{z^{\prime \prime} s}\right) J^{k}\left(\underline{x}_{10}, x_{32}^{2}, z^{\prime \prime} s\right) .
\end{gathered}
$$

In arriving at eqs. (2.34) we have neglected terms like $I^{k}\left(\underline{x}_{21}, z^{\prime} s\right)$, which are zero after the angular integration over the directions of $\underline{x}_{21}$.

The inhomogeneous terms in eqs. (2.34) are

$$
I^{(0) k}\left(\underline{x}_{10}, z s\right)=\int d^{2} x_{1} x_{1}^{k} G_{10}^{(0)}(z s),
$$

where, again, the integration is performed with fixed $\underline{x}_{10}$. The Born-level initial conditions for the polarized dipole amplitude are (see eq. (13a) in [3], which assumes the polarized 
target to be a single quark at the origin $\underline{0}$ in the transverse plane)

$$
G_{10}^{(0)}(z s)=\frac{\alpha_{s}^{2} C_{F}}{2 N_{c}}\left[\frac{C_{F}}{x_{1}^{2}}-2 \pi \delta^{2}\left(\underline{x}_{1}\right) \ln \left(z s x_{10}^{2}\right)\right] .
$$

Using eq. (2.36) in eq. (2.35) while assuming that the $\underline{x}_{1}$-integral is regulated in the IR by an upper cutoff on the magnitude of $\underline{x}_{1}$ yields

$$
I^{(0) k}\left(\underline{x}_{10}, z s\right)=\int d^{2} x_{1} x_{1}^{k} \theta\left(\frac{1}{\Lambda}-x_{1}\right) \frac{\alpha_{s}^{2} C_{F}}{2 N_{c}}\left[\frac{C_{F}}{x_{1}^{2}}-2 \pi \delta^{2}\left(\underline{x}_{1}\right) \ln \left(z s x_{10}^{2}\right)\right]=0 .
$$

With the zero inhomogeneous terms, eqs. (2.34) have a trivial solution:

$$
I^{k}\left(\underline{x}_{10}, z s\right)=0, \quad J^{k}\left(\underline{x}_{10}, x_{21}^{2}, z s\right)=0 .
$$

However, this conclusion changes for a slight variation of the IR regularization in eq. (2.37). For instance, using $\theta\left[\frac{1}{\Lambda}-\left(\underline{x}_{1}+\underline{x}_{0}\right) / 2\right]$ gives a non-zero result,

$$
I^{(0) k}\left(\underline{x}_{10}, z s\right)=\int d^{2} x_{1} x_{1}^{k} \theta\left(\frac{1}{\Lambda}-\frac{\underline{x}_{1}+\underline{x}_{0}}{2}\right) \frac{\alpha_{s}^{2} C_{F}}{2 N_{c}}\left[\frac{C_{F}}{x_{1}^{2}}-2 \pi \delta^{2}\left(\underline{x}_{1}\right) \ln \left(z s x_{10}^{2}\right)\right]=\frac{\alpha_{s}^{2} \pi C_{F}^{2}}{4 N_{c}} x_{10}^{k} .
$$

Therefore, we will proceed assuming that the inhomogeneous term $I^{(0) k}\left(\underline{x}_{10}, z s\right)$ is not zero. As we will shortly see, the leading small- $x$ asymptotics of $L_{q+\bar{q}}\left(x, Q^{2}\right)$ is independent of whether $I^{(0) k}\left(\underline{x}_{10}, z s\right)$ is zero or not.

Using the fact that neither the initial condition (2.35) nor the evolution equations (2.33) contain a two-dimensional Levi-Civita symbol $\epsilon^{i j}$, we can write, without any loss of generality,

$$
\begin{aligned}
I^{k}\left(\underline{x}_{10}, z s\right) & =x_{10}^{k} I\left(x_{10}^{2}, z s\right), \\
J^{k}\left(\underline{x}_{10}, x_{21}^{2}, z s\right) & =x_{10}^{k} J\left(x_{10}^{2}, x_{21}^{2}, z s\right) .
\end{aligned}
$$

Substituting eqs. (2.40) into eqs. (2.34) yields

$$
\begin{aligned}
I\left(x_{10}^{2}, z s\right) & =I^{(0)}\left(x_{10}^{2}, z s\right)+\frac{\alpha_{s} N_{c}}{2 \pi} \int_{\frac{1}{x_{10}^{2}}}^{z} \frac{d z^{\prime}}{z^{\prime}} \int_{\frac{1}{z^{\prime} s}}^{x_{10}^{2}} \frac{d x_{21}^{2}}{x_{21}^{2}} J\left(x_{10}^{2}, x_{21}^{2}, z^{\prime} s\right), \\
J\left(x_{10}^{2}, x_{21}^{2}, z^{\prime} s\right) & =I^{(0)}\left(x_{10}^{2}, z^{\prime} s\right)+\frac{\alpha_{s} N_{c}}{2 \pi} \int_{\frac{1}{x_{10}^{2}}}^{z^{\prime}} \frac{d z^{\prime \prime}}{z^{\prime \prime}} \int_{\frac{1}{z^{\prime \prime} s}}^{\min \left\{x_{10}^{2}, x_{21}^{2}\left(z^{\prime} / z^{\prime \prime}\right)\right\}} \frac{d x_{32}^{2}}{x_{32}^{2}} J\left(x_{10}^{2}, x_{32}^{2}, z^{\prime \prime} s\right) .
\end{aligned}
$$

Inspired by eq. (2.39) and by the prior experience $[2,4,5]$, which demonstrated independence of small- $x$ asymptotics on the inhomogeneous term for helicity distributions, let us assume that $I^{(0)}\left(x_{10}^{2}, z s\right)=I^{(0)}\left(x_{10}^{2}\right)$. In this case, defining

$$
\bar{I}\left(x_{10}^{2}, z s\right)=\frac{I\left(x_{10}^{2}, z s\right)}{I^{(0)}\left(x_{10}^{2}\right)}, \quad \bar{J}\left(x_{10}^{2}, x_{21}^{2}, z^{\prime} s\right)=\frac{J\left(x_{10}^{2}, x_{21}^{2}, z^{\prime} s\right)}{I^{(0)}\left(x_{10}^{2}\right)},
$$


we reduce eqs. (2.41) to

$$
\begin{gathered}
\bar{I}\left(x_{10}^{2}, z s\right)=1+\frac{\alpha_{s} N_{c}}{2 \pi} \int_{\frac{1}{x_{10}^{2}}}^{z} \frac{d z^{\prime}}{z^{\prime}} \int_{\frac{1}{z^{\prime} s}}^{x_{10}^{2}} \frac{d x_{21}^{2}}{x_{21}^{2}} \bar{J}\left(x_{10}^{2}, x_{21}^{2}, z^{\prime} s\right) \\
\bar{J}\left(x_{10}^{2}, x_{21}^{2}, z^{\prime} s\right)=1+\frac{\alpha_{s} N_{c}}{2 \pi} \int_{\frac{1}{x_{10}^{2}}}^{z^{\prime}} \frac{d z^{\prime \prime}}{z^{\prime \prime}} \int_{\frac{1}{z^{\prime \prime} s}}^{\min \left\{x_{10}^{2}, x_{21}^{2}\left(z^{\prime} / z^{\prime \prime}\right)\right\}} \frac{d x_{32}^{2}}{x_{32}^{2}} \bar{J}\left(x_{10}^{2}, x_{32}^{2}, z^{\prime \prime} s\right) .
\end{gathered}
$$

These equations are solved in appendix B (with $\bar{I}=\bar{G}_{5}$ and $\bar{J}=\bar{\Gamma}_{5}$ there, and with the $\beta=+1$ case of the solution in appendix B being of interest to us here). The resulting leading high-energy contribution is (cf. eq. (B.15))

$$
\bar{I}\left(x_{10}^{2}, z\right)=\frac{I_{1}\left(2 \sqrt{\frac{\alpha_{s} N_{c}}{2 \pi}} \ln \left(z s x_{10}^{2}\right)\right)}{\sqrt{\frac{\alpha_{s} N_{c}}{2 \pi}} \ln \left(z s x_{10}^{2}\right)} .
$$

We conclude that, for $z s x_{10}^{2} \gg 1$,

$$
I\left(x_{10}^{2}, z\right)=I^{(0)}\left(x_{10}^{2}, z\right) \frac{I_{1}\left(2 \sqrt{\frac{\alpha_{s} N_{c}}{2 \pi}} \ln \left(z s x_{10}^{2}\right)\right)}{\sqrt{\frac{\alpha_{s} N_{c}}{2 \pi}} \ln \left(z s x_{10}^{2}\right)} \sim\left(z s x_{10}^{2}\right)^{2 \sqrt{\frac{\alpha_{s} N_{c}}{2 \pi}}} .
$$

\subsection{Quark OAM distribution at small $x$}

Employing eqs. (2.32a) and (2.40a) in eq. (2.27) we rewrite the quark OAM distribution as $L_{q+\bar{q}}\left(x, Q^{2}\right)=\frac{8 N_{c}}{(2 \pi)^{5}} \int d^{2} k_{\perp} d^{2} x_{10} e^{i \underline{k} \cdot \underline{x}_{10}} \frac{\left(\underline{x}_{10} \times \underline{k}\right)^{2}}{x_{10}^{2} \underline{k}^{2}} \int_{\Lambda^{2} / s}^{1} \frac{d z}{z} I\left(x_{10}^{2}, z s\right)-\sum_{f}\left[\Delta q^{f}\left(x, Q^{2}\right)+\Delta \bar{q}^{f}\left(x, Q^{2}\right)\right]$.

Equation (2.45) allows us to conclude that the first term on the right-hand side of eq. (2.46) has the following small- $x$ asymptotics:

$$
\frac{8 N_{c}}{(2 \pi)^{5}} \int d^{2} k_{\perp} d^{2} x_{10} e^{i \underline{k} \cdot \underline{x}_{10}} \frac{\left(\underline{x}_{10} \times \underline{k}\right)^{2}}{x_{10}^{2} \underline{k}^{2}} \int_{\Lambda^{2} / s}^{1} \frac{d z}{z} I\left(x_{10}^{2}, z s\right) \sim\left(\frac{1}{x}\right)^{2 \sqrt{\frac{\alpha_{s} N_{c}}{2 \pi}}} .
$$

At the same time, the small- $x$ asymptotics of the quark helicity distribution was found in $[2,4]$ to be

$$
\Delta \Sigma\left(x, Q^{2}\right)=\sum_{f}\left[\Delta q^{f}\left(x, Q^{2}\right)+\Delta \bar{q}^{f}\left(x, Q^{2}\right)\right] \sim\left(\frac{1}{x}\right)^{\alpha_{h}^{q}}=\left(\frac{1}{x}\right)^{\frac{4}{\sqrt{3}} \sqrt{\frac{\alpha_{s} N_{c}}{2 \pi}}} \approx\left(\frac{1}{x}\right)^{2.31 \sqrt{\frac{\alpha_{s} N_{c}}{2 \pi}}}
$$


in the same DLA limit. Since $4 / \sqrt{3}>2$, we conclude that at small- $x$ the second term on the right-hand side of eq. (2.46) dominates. Dropping the first term we arrive at

$$
L_{q+\bar{q}}\left(x, Q^{2}\right) \approx-\sum_{f}\left[\Delta q^{f}\left(x, Q^{2}\right)+\Delta \bar{q}^{f}\left(x, Q^{2}\right)\right]=-\Delta \Sigma\left(x, Q^{2}\right) .
$$

This result is in agreement with eq. (40) of [16], if we assume that $c=\mathcal{O}\left(\sqrt{\alpha_{s}}\right) \ll 1$ in it. Note, however, that the results in section IV of [16] (including eq. (40) there) were derived under the assumption that $|\Delta G| \gg|\Delta \Sigma|$ at small $x$, which is the opposite of what was found at DLA in $[2,4,5]$.

The small- $x$ asymptotics of the quark OAM easily follows from eqs. (2.49) and (2.48). We conclude that

$$
L_{q+\bar{q}}\left(x, Q^{2}\right)=-\Delta \Sigma\left(x, Q^{2}\right) \sim\left(\frac{1}{x}\right)^{\frac{4}{\sqrt{3}} \sqrt{\frac{\alpha_{s} N_{c}}{2 \pi}}}
$$

at small $x$ and in the large- $N_{c}$ limit (assuming gluon dominance in the latter). Note that the net small- $x$ quark contribution to the proton spin is

$$
\frac{1}{2} \Delta \Sigma\left(x, Q^{2}\right)+L_{q+\bar{q}}\left(x, Q^{2}\right)=-\frac{1}{2} \Delta \Sigma\left(x, Q^{2}\right)
$$

and is, therefore, non-zero.

\section{Gluon OAM}

\subsection{The gluon OAM operator}

Now we turn our attention to the gluon OAM distribution. First we need to construct the corresponding operator, and simplify it at small $x$. Again we begin with the definition of the OAM in terms of the Wigner function given in eq. (2.1). We need to obtain the gluon Wigner distribution.

Similar to the quark case, to construct the gluon Wigner function we first consider the unpolarized dipole gluon TMD in a longitudinally polarized proton $[49,53]$,

$f_{1}^{G \operatorname{dip}}\left(x, k_{T}^{2}\right)=\frac{2}{x P^{+}} \int \frac{d \xi^{-} d^{2} \xi}{(2 \pi)^{3}} e^{i x P^{+} \xi^{-}-i \underline{k} \cdot \underline{\xi}}\left\langle P, S_{L}\left|\operatorname{tr}\left[F^{+i}(0) \mathcal{U}^{[+]}[0, \xi] F^{+i}(\xi) \mathcal{U}^{[-]}[\xi, 0]\right]\right| P, S_{L}\right\rangle_{\xi^{+}=0}$,

where the future- and past-pointing Wilson line staples are $\mathcal{U}^{[+]}[0, \xi]=V_{0}\left[0^{-},+\infty\right] V_{\xi}\left[+\infty, \xi^{-}\right]$ and $\mathcal{U}^{[-]}[\xi, 0]=V_{\xi}\left[\xi^{-},-\infty\right] V_{\underline{0}}\left[-\infty, 0^{-}\right]$in $A^{-}=0$ gauge. To extract the gluon Wigner distribution we employ the CGC averaging in eq. (A.2) to write

$$
\begin{aligned}
f_{1}^{G \operatorname{dip}}\left(x, k_{T}^{2}\right) & =\frac{4}{x} \frac{1}{(2 \pi)^{3}} \int d \xi^{-} d^{2} \xi_{\perp} d b^{-} d^{2} b_{\perp} e^{i x P^{+} \xi^{-}-i k \underline{\xi} \underline{\xi}}\left\langle\operatorname{tr}\left[F^{+i}(b) \mathcal{U}^{[+]}[b, b+\xi] F^{+i}(b+\xi) \mathcal{U}^{[-]}[b+\xi, b]\right]\right\rangle \\
& \equiv \frac{P^{+}}{(2 \pi)^{3}} \int d^{2}\left(b_{\perp}+\frac{1}{2} \xi_{\perp}\right) d\left(b^{-}+\frac{1}{2} \xi^{-}\right) W^{G \operatorname{dip}}\left(k, b+\frac{1}{2} \xi\right) .
\end{aligned}
$$


(The factor of $P^{+}$is to ensure that the gluon PDF is per $d x$, not $d k^{+}$.) We read off the unpolarized gluon dipole Wigner distribution

$$
\begin{aligned}
W^{G \operatorname{dip}}(k, b)= & \frac{4}{x P^{+}} \int d \xi^{-} d^{2} \xi \perp e^{i x P^{+} \xi^{-}-i \underline{k} \cdot \underline{\xi}} \\
& \times\left\langle\operatorname{tr}\left[F^{+i}\left(b-\frac{1}{2} \xi\right) \mathcal{U}^{[+]}\left[b-\frac{1}{2} \xi, b+\frac{1}{2} \xi\right] F^{+i}\left(b+\frac{1}{2} \xi\right) \mathcal{U}^{[-]}\left[b+\frac{1}{2} \xi, b-\frac{1}{2} \xi\right]\right]\right\rangle .
\end{aligned}
$$

Using it in (2.1) we arrive at the gluon dipole OAM definition

$$
\begin{aligned}
L_{G}\left(Q^{2}\right)= & \frac{4}{(2 \pi)^{3}} \int d^{2} b_{\perp} d b^{-} d^{2} k_{\perp} \frac{d x}{x} d \xi^{-} d^{2} \xi_{\perp}(\underline{b} \times \underline{k}) e^{i x P^{+} \xi^{-}-i \underline{k} \cdot \underline{\xi}} \\
& \times\left\langle\operatorname{tr}\left[F^{+i}\left(b-\frac{1}{2} \xi\right) \mathcal{U}^{[+]}\left[b-\frac{1}{2} \xi, b+\frac{1}{2} \xi\right] F^{+i}\left(b+\frac{1}{2} \xi\right) \mathcal{U}^{[-]}\left[b+\frac{1}{2} \xi, b-\frac{1}{2} \xi\right]\right]\right\rangle .
\end{aligned}
$$

In appendix $\mathrm{C}$ we show that this definition of gluon OAM is consistent with the standard Jaffe-Manohar gluon OAM definition [7].

Just like for quark OAM, we are interested in the gluon OAM distribution $L_{G}\left(x, Q^{2}\right)=$ $d L_{G}\left(Q^{2}\right) / d x$, which is given by

$$
\begin{aligned}
L_{G}\left(x, Q^{2}\right)= & \frac{4}{(2 \pi)^{3} x} \int d^{2} b_{\perp} d b^{-} d^{2} k_{\perp} d \xi^{-} d^{2} \xi_{\perp}(\underline{b} \times \underline{k}) e^{i x P^{+} \xi^{-}-i \underline{k} \cdot \underline{\xi}} \\
& \times\left\langle\operatorname{tr}\left[F^{+i}\left(b-\frac{1}{2} \xi\right) \mathcal{U}^{[+]}\left[b-\frac{1}{2} \xi, b+\frac{1}{2} \xi\right] F^{+i}\left(b+\frac{1}{2} \xi\right) \mathcal{U}^{[-]}\left[b+\frac{1}{2} \xi, b-\frac{1}{2} \xi\right]\right]\right\rangle .
\end{aligned}
$$

The presence of $\epsilon^{i j}$ in $\underline{b} \times \underline{k}$ of eq. (3.5) demands that there has to be another $\epsilon^{i j}$ in the angle brackets $\langle\ldots\rangle$, thus eliminating the contributions of the standard (unpolarized) BFKL/BK/JIMWLK evolution. This is similar to the case of gluon helicity [5].

\subsection{Evaluation of the gluon OAM operator at small $x$}

Our next steps are to simplify the gluon OAM operator definition (3.4) along the lines of $[5,18]$ and evolve it to small $x$. In $A^{-}=0$ gauge eq. (3.5) becomes

$$
\begin{aligned}
L_{G}\left(x, Q^{2}\right)= & \frac{4}{(2 \pi)^{3} x} \int d \xi^{-} d^{2} \xi_{\perp} d \zeta^{-} d^{2} \zeta_{\perp} d^{2} k_{\perp}\left(\frac{\underline{\zeta}+\underline{\xi}}{2} \times \underline{k}\right) e^{i x P^{+}\left(\xi^{-}-\zeta^{-}\right)-i \underline{k} \cdot(\underline{\xi}-\underline{\zeta})} \\
& \times\left\langle\operatorname{tr}\left[V_{\underline{\zeta}}\left[-\infty, \zeta^{-}\right] F^{+i}(\zeta) V_{\underline{\zeta}}\left[\zeta^{-},+\infty\right] V_{\underline{\xi}}\left[+\infty, \xi^{-}\right] F^{+i}(\xi) V_{\underline{\xi}}\left[\xi^{-},-\infty\right]\right]\right\rangle,
\end{aligned}
$$

where we have also changed variables from $b \mp \frac{1}{2} \xi \rightarrow \zeta, \xi$.

For the unpolarized gluon distribution, it is sufficient to replace the field-strength tensors by their eikonal approximations, $F^{+i} \approx-\partial_{\perp}^{i} A^{+}$: however, in eq. (3.6) this would give zero since the eikonal approximation contains no $\epsilon^{i j}$ needed to obtain a non-zero result. Hence we need to look for a sub-eikonal gluon field which (for mass-independent terms) depends on the polarization of the target proton, which would bring another $\epsilon^{i j}$. Proton 
polarization dependence enters through the sub-eikonal gluon field $A^{i}$ with $i=1,2$. The situation is similar to the case of gluon helicity at small $x$ [5]. We expand the product of field-strength tensors to the first non-vanishing sub-eikonal order, that is, to the linear order in $A^{i}$, getting

$$
\begin{aligned}
F^{+i}(\zeta) \cdots F^{+j}(\xi) & =\left(\partial^{+} A_{\perp}^{i}(\zeta)-\partial^{i} A^{+}(\zeta)-i g\left[A^{+}(\zeta), A_{\perp}^{i}(\zeta)\right]\right) \cdots\left(\partial^{+} A_{\perp}^{j}(\xi)-\partial^{j} A^{+}(\xi)-i g\left[A^{+}(\xi), A_{\perp}^{j}(\xi)\right]\right) \\
& \approx\left(\frac{\partial}{\partial \zeta^{-}} A_{\perp}^{i}(\zeta)-i g\left[A^{+}(\zeta), A_{\perp}^{i}(\zeta)\right]\right) \cdots\left(\frac{\partial}{\partial \xi_{\perp}^{j}} A^{+}(\xi)\right)+\left(\frac{\partial}{\partial \zeta_{\perp}^{i}} A^{+}(\zeta)\right) \cdots\left(\frac{\partial}{\partial \xi^{-}} A_{\perp}^{j}(\xi)-i g\left[A^{+}(\xi), A_{\perp}^{j}(\xi)\right]\right)
\end{aligned}
$$

We next convert the sub-eikonal part of the field-strength tensor $F^{+i}(\zeta)$ into a total derivative,

$$
V_{\underline{\zeta}}\left[-\infty, \zeta^{-}\right]\left(\frac{\partial}{\partial \zeta^{-}} A_{\perp}^{i}(\zeta)-i g\left[A^{+}(\zeta), A_{\perp}^{i}(\zeta)\right]\right) V_{\underline{\zeta}}\left[\zeta^{-},+\infty\right]=\frac{\partial}{\partial \zeta^{-}}\left(V_{\underline{\zeta}}\left[-\infty, \zeta^{-}\right] A_{\perp}^{i}(\zeta) V_{\underline{\zeta}}\left[\zeta^{-},+\infty\right]\right),
$$

which, after integration by parts, acts on the Fourier factor and generates a net factor of $+i x P^{+}$on the right of eq. (3.6). Analogously, the sub-eikonal part of the $F^{+j}(\xi)$ fieldstrength tensor gives a net factor of $-i x P^{+}$and the operator $A_{\perp}^{j}(\xi)$. After taking these derivatives, we set $e^{i x P^{+}\left(\xi^{-}-\zeta^{-}\right)} \approx 1$ in eq. (3.6) (thus neglecting higher powers of $x \ll 1$ ). We arrive at

$$
\begin{aligned}
L_{G}\left(x, Q^{2}\right)=\frac{4 i P^{+}}{(2 \pi)^{3}} & \int d \xi^{-} d^{2} \xi_{\perp} d \zeta^{-} d^{2} \zeta_{\perp} d^{2} k_{\perp}\left(\frac{\underline{\zeta}+\underline{\xi}}{2} \times \underline{k}\right) e^{-i \underline{k} \cdot(\underline{\xi}-\underline{\zeta})} \\
\times & \left\{\left\langle\operatorname{tr}\left[V_{\underline{\underline{\zeta}}}\left[-\infty, \zeta^{-}\right] A^{i}(\zeta) V_{\underline{\xi}}\left[\zeta^{-},+\infty\right] V_{\underline{\xi}}\left[+\infty, \xi^{-}\right]\left(\frac{\partial}{\partial \xi_{\perp}^{i}} A^{+}(\xi)\right) V_{\underline{\xi}}\left[\xi^{-},-\infty\right]\right]\right\rangle\right. \\
& \left.-\left\langle\operatorname{tr}\left[V_{\underline{\zeta}}\left[-\infty, \zeta^{-}\right]\left(\frac{\partial}{\partial \zeta_{\perp}^{i}} A^{+}(\zeta)\right) V_{\underline{\zeta}}\left[\zeta^{-},+\infty\right] V_{\underline{\xi}}\left[+\infty, \xi^{-}\right] A^{i}(\xi) V_{\underline{\xi}}\left[\xi^{-},-\infty\right]\right]\right\rangle\right\} .
\end{aligned}
$$

Further, writing

$$
\int_{-\infty}^{\infty} d \zeta^{-} V_{\underline{\zeta}}\left[-\infty, \zeta^{-}\right]\left(\frac{\partial}{\partial \zeta_{\perp}^{i}} A^{+}(\zeta)\right) V_{\underline{\zeta}}\left[\zeta^{-},+\infty\right]=\frac{i}{g} \frac{\partial}{\partial \zeta_{\perp}^{i}} V_{\underline{\zeta}}[-\infty,+\infty],
$$

yields

$$
\begin{aligned}
L_{G}\left(x, Q^{2}\right)=\frac{4 P^{+}}{g(2 \pi)^{3}} & \int d^{2} \xi_{\perp} d^{2} \zeta_{\perp} d^{2} k_{\perp}\left(\frac{\underline{\zeta}+\underline{\xi}}{2} \times \underline{k}\right) e^{-i \underline{k} \cdot(\underline{\xi}-\underline{\zeta})} \\
& \times\left\{\left\langle\operatorname{tr}\left[\int d \zeta^{-} V_{\underline{\zeta}}\left[-\infty, \zeta^{-}\right] A^{i}(\zeta) V_{\underline{\zeta}}\left[\zeta^{-},+\infty\right] \frac{\partial}{\partial \xi_{\perp}^{i}} V_{\underline{\xi}}[+\infty,-\infty]\right]\right\rangle\right. \\
& \left.+\left\langle\operatorname{tr}\left[\frac{\partial}{\partial \zeta_{\perp}^{i}} V_{\underline{\zeta}}[-\infty,+\infty] \int d \xi^{-} V_{\underline{\xi}}\left[+\infty, \xi^{-}\right] A^{i}(\xi) V_{\underline{\xi}}\left[\xi^{-},-\infty\right]\right]\right\rangle\right\} .
\end{aligned}
$$


Integrating by parts we obtain

$$
\begin{aligned}
L_{G}\left(x, Q^{2}\right)=\frac{4 P^{+}}{g(2 \pi)^{3}} & \int d^{2} \xi_{\perp} d^{2} \zeta_{\perp} d^{2} k_{\perp} e^{-i \underline{k} \cdot(\underline{\xi}-\underline{\zeta})} \\
\times & \left\{i k^{i}\left(\frac{\underline{\zeta}+\underline{\xi}}{2} \times \underline{k}\right)\left\langle\operatorname{tr}\left[\int d \zeta^{-} V_{\underline{\xi}}\left[-\infty, \zeta^{-}\right] A^{i}(\zeta) V_{\underline{\zeta}}\left[\zeta^{-},+\infty\right] V_{\underline{\xi}}[+\infty,-\infty]\right]\right\rangle\right. \\
& -i k^{i}\left(\frac{\underline{\zeta}+\underline{\xi}}{2} \times \underline{k}\right)\left\langle\operatorname{tr}\left[V_{\underline{\zeta}}[-\infty,+\infty] \int d \xi^{-} V_{\underline{\xi}}\left[+\infty, \xi^{-}\right] A^{i}(\xi) V_{\underline{\xi}}\left[\xi^{-},-\infty\right]\right]\right\rangle \\
& -\frac{1}{2} \epsilon^{i j} k^{j}\left\langle\operatorname{tr}\left[\int d \zeta^{-} V_{\underline{\xi}}\left[-\infty, \zeta^{-}\right] A^{i}(\zeta) V_{\underline{\zeta}}\left[\zeta^{-},+\infty\right] V_{\underline{\xi}}[+\infty,-\infty]\right]\right\rangle \\
& \left.-\frac{1}{2} \epsilon^{i j} k^{j}\left\langle\operatorname{tr}\left[V_{\underline{\zeta}}[-\infty,+\infty] \int d \xi^{-} V_{\underline{\xi}}\left[+\infty, \xi^{-}\right] A^{i}(\xi) V_{\underline{\xi}}\left[\xi^{-},-\infty\right]\right]\right\rangle\right\} .
\end{aligned}
$$

Define the polarized Wilson line [5, 18]

$$
\begin{aligned}
\left(V_{\underline{x}}^{\mathrm{pol}}\right)_{\perp}^{i} & \equiv \int_{-\infty}^{+\infty} d x^{-} V_{\underline{x}}\left[+\infty, x^{-}\right]\left(i g P^{+} A_{\perp}^{i}(x)\right) V_{\underline{x}}\left[x^{-},-\infty\right] \\
& =\frac{1}{2} \int_{-\infty}^{+\infty} d x^{-} V_{\underline{x}}\left[+\infty, x^{-}\right]\left(i g \bar{A}_{\perp}^{i}(x)\right) V_{\underline{x}}\left[x^{-},-\infty\right] .
\end{aligned}
$$

One may call it the polarized Wilson line of the second kind to stress its difference from a similar, but distinct, object defined for quark helicity and OAM (see also [6]). With the help of eq. (3.13) we write

$$
\begin{aligned}
L_{G}\left(x, Q^{2}\right)= & \frac{4}{g^{2}(2 \pi)^{3}} \int d^{2} \xi_{\perp} d^{2} \zeta_{\perp} d^{2} k_{\perp} d x e^{-i \underline{k} \cdot(\underline{\xi}-\underline{\zeta})} \\
& \times\left\{-k^{i}\left(\frac{\underline{\zeta}+\underline{\xi}}{2} \times \underline{k}\right)\left\langle\operatorname{tr}\left[\left(V_{\underline{\underline{k}}}^{\mathrm{pol} \dagger}\right)_{\perp}^{i} V_{\underline{\xi}}[+\infty,-\infty]\right]\right\rangle-k^{i}\left(\frac{\underline{\zeta}+\underline{\xi}}{2} \times \underline{k}\right)\left\langle\operatorname{tr}\left[V_{\underline{\zeta}}[-\infty,+\infty]\left(V_{\underline{\xi}}^{\mathrm{pol}}\right)_{\perp}^{i}\right]\right\rangle\right. \\
& \left.-\frac{i}{2} \epsilon^{i j} k^{j}\left\langle\operatorname{tr}\left[\left(V_{\underline{\underline{\xi}}}^{\mathrm{pol} \dagger}\right)_{\perp}^{i} V_{\underline{\xi}}[+\infty,-\infty]\right]\right\rangle+\frac{i}{2} \epsilon^{i j} k^{j}\left\langle\operatorname{tr}\left[V_{\underline{\zeta}}[-\infty,+\infty]\left(V_{\underline{\xi}}^{\mathrm{pol}}\right)_{\perp}^{i}\right]\right\rangle\right\} .
\end{aligned}
$$

Swapping $\underline{\zeta} \leftrightarrow \underline{\xi}$ in the second and fourth terms in the curly brackets along with replacing $\underline{k} \rightarrow-\underline{k}$ for those terms we get

$$
\begin{aligned}
L_{G}\left(x, Q^{2}\right)= & -\frac{4}{g^{2}(2 \pi)^{3}} \int d^{2} \xi_{\perp} d^{2} \zeta_{\perp} d^{2} k_{\perp} d x e^{-i \underline{k} \cdot(\underline{\xi}-\underline{\zeta})} \\
& \times\left\{k^{i}\left(\frac{\underline{\zeta}+\underline{\xi}}{2} \times \underline{k}\right)\left\langle\operatorname{tr}\left[\left(V_{\underline{\underline{\xi}}}^{\mathrm{pol} \dagger}\right)_{\perp}^{i} V_{\underline{\xi}}[+\infty,-\infty]\right]\right\rangle+k^{i}\left(\frac{\underline{\zeta}+\underline{\xi}}{2} \times \underline{k}\right)\left\langle\operatorname{tr}\left[V_{\underline{\xi}}[-\infty,+\infty]\left(V_{\underline{\underline{\xi}}}^{\mathrm{pol}}\right)_{\perp}^{i}\right]\right\rangle\right. \\
& \left.+\frac{i}{2} \epsilon^{i j} k^{j}\left\langle\operatorname{tr}\left[\left(V_{\underline{\underline{\zeta}}}^{\mathrm{pol} \dagger}\right)_{\perp}^{i} V_{\underline{\xi}}[+\infty,-\infty]\right]\right\rangle+\frac{i}{2} \epsilon^{i j} k^{j}\left\langle\operatorname{tr}\left[V_{\underline{\xi}}[-\infty,+\infty]\left(V_{\underline{\underline{\zeta}}}^{\mathrm{pol}}\right)_{\perp}^{i}\right]\right\rangle\right\} .
\end{aligned}
$$


Defining another polarized dipole-like operator [5]

$$
G_{10}^{i}(z s) \equiv \frac{1}{2 N_{c}}\left\langle\operatorname{tr}\left[V_{\underline{0}}\left(V_{\underline{1}}^{\mathrm{pol} \dagger}\right)_{\perp}^{i}\right]+\text { c.c. }\right\rangle(z s)
$$

and employing a more conventional (at small $x$ ) notation we rewrite eq. (3.15) as

$$
L_{G}\left(x, Q^{2}\right)=-\frac{8 N_{c}}{g^{2}(2 \pi)^{3}} \int d^{2} x_{1} d^{2} x_{0} d^{2} k_{\perp} e^{i \underline{k} \cdot \underline{x}_{10}}\left[k^{i}\left(\frac{\underline{x}_{1}+\underline{x}_{0}}{2} \times \underline{k}\right)+\frac{i}{2} \epsilon^{i j} k^{j}\right] G_{10}^{i}\left(z s=\frac{Q^{2}}{x}\right) .
$$

Comparing this with the dipole gluon helicity TMD at small $x[5]$

$$
g_{1 L}^{G \operatorname{dip}}\left(x, k_{T}^{2}\right)=\frac{-8 i N_{c}}{g^{2}(2 \pi)^{3}} \int d^{2} x_{1} d^{2} x_{0} e^{i k \underline{k}_{10}} k_{\perp}^{i} \epsilon^{i j} G_{10}^{j}\left(z s=\frac{Q^{2}}{x}\right)
$$

we recast eq. (3.17) as

$L_{G}\left(x, Q^{2}\right)=-\frac{8 N_{c}}{g^{2}(2 \pi)^{3}} \int d^{2} x_{1} d^{2} x_{0} d^{2} k_{\perp} e^{i \underline{k} \cdot \underline{x}_{10}} k^{i}\left(\frac{\underline{x}_{1}+\underline{x}_{0}}{2} \times \underline{k}\right) G_{10}^{i}\left(z s=\frac{Q^{2}}{x}\right)-\frac{1}{2} \Delta G\left(x, Q^{2}\right)$,

where

$$
\Delta G\left(x, Q^{2}\right)=\int d^{2} k_{\perp} g_{1 L}^{G \operatorname{dip}}\left(x, k_{T}^{2}\right)
$$

Next, write $k^{i}=-i \nabla_{x_{1}}^{i}$ and integrate by parts. This yields

$L_{G}\left(x, Q^{2}\right)=-\frac{8 N_{c}}{g^{2}(2 \pi)^{3}} \int d^{2} x_{1} d^{2} x_{0} d^{2} k_{\perp} e^{i \underline{k} \underline{x}_{10}}\left[\frac{i}{2} \epsilon^{i j} k^{j} G_{10}^{i}+\left(\frac{\underline{x}_{1}+\underline{x}_{0}}{2} \times \underline{k}\right) i \nabla_{x_{1}}^{i} G_{10}^{i}\right]-\frac{1}{2} \Delta G\left(x, Q^{2}\right)$,

where we suppress the argument of $G_{10}^{i}$ for brevity. Using eq. (3.18) again we arrive at

$$
L_{G}\left(x, Q^{2}\right)=-\frac{8 N_{c}}{g^{2}(2 \pi)^{3}} \int d^{2} x_{1} d^{2} x_{0} d^{2} k_{\perp} e^{i \underline{k} \underline{x}_{10}}\left(\frac{\underline{x}_{1}+\underline{x}_{0}}{2} \times \underline{k}\right) i \nabla_{x_{1}}^{i} G_{10}^{i}-\Delta G\left(x, Q^{2}\right) .
$$

Taking the Born-level $G_{10}^{i}$ from eq. (92) of [5] calculated for a single polarized quark target at $\underline{0}$,

$$
G_{10}^{i(0)}(z)=-\frac{\alpha_{s}^{2} C_{F}}{N_{c}} \epsilon^{i j} \frac{\left(\underline{x}_{1}-\underline{b}\right)^{j}}{\left|\underline{x}_{1}-\underline{b}\right|^{2}} \ln \frac{\left|\underline{x}_{1}-\underline{b}\right|}{\left|\underline{x}_{0}-\underline{b}\right|}
$$

we get $\nabla_{x_{1}}^{i} G_{10}^{i}=0$. From eq. (3.22) we see that at this Born level $L_{G}\left(x, Q^{2}\right)=-\Delta G\left(x, Q^{2}\right)$, in agreement with eq. (50) of [18] (after the latter is corrected by a factor of 2 , as clarified in footnote 7 of [16]). This result appears to be similar to the parton model argument in appendix B of [18]. As we will see below, the Born level $L_{G}\left(x, Q^{2}\right)=-\Delta G\left(x, Q^{2}\right)$ relation does not appear to survive the DLA evolution. 
In the quark OAM case worked out above we learned that it is easier to work with the polarized dipole amplitude weighed by the position of the polarized quark $\underline{x}_{1}$ and then integrated over all $\underline{x}_{1}$, as opposed to using other weight factors (e.g. $\underline{x}_{0}$ or $\left(\underline{x}_{1}+\underline{x}_{0}\right) / 2$ as in eq. (3.22)). To obtain the gluon OAM in terms of $x_{1}$-weighed polarized dipole amplitude, start with eq. (3.19) and write $\frac{\underline{x}_{1}+\underline{x}_{0}}{2}=\underline{x}_{1}-\frac{1}{2} \underline{x}_{10}$ along with replacing $d^{2} x_{1} d^{2} x_{0} \rightarrow$ $d^{2} x_{1} d^{2} x_{10}$. Then further replacing $\underline{x}_{10} \rightarrow-i \underline{\nabla}_{k}$ and integrating over $\underline{k}$ by parts one arrives at

$$
L_{G}\left(x, Q^{2}\right)=-\frac{8 N_{c}}{g^{2}(2 \pi)^{3}} \int d^{2} x_{1} d^{2} x_{10} d^{2} k_{\perp} e^{i \underline{k} \underline{x}_{10}} k^{i}\left(\underline{x}_{1} \times \underline{k}\right) G_{10}^{i}\left(z s=\frac{Q^{2}}{x}\right) .
$$

This appears to be the most compact expression for the gluon OAM at small $x$. It also suggests that in the polarized dipole 01 the two transverse coordinates do not enter on equal footing: this is indeed natural, since in eq. (3.16) line 1 is polarized, while line 0 is not.

Further, we replace $k^{i} \rightarrow-i \nabla_{10}^{i}$, and, integrating by parts obtain

$$
L_{G}\left(x, Q^{2}\right)=-\frac{8 i N_{c}}{g^{2}(2 \pi)^{3}} \int d^{2} x_{1} d^{2} x_{10} d^{2} k_{\perp} e^{i \underline{k} \underline{x}_{10}}\left(\underline{x}_{1} \times \underline{k}\right) \nabla_{10}^{i} G_{10}^{i}\left(z s=\frac{Q^{2}}{x}\right) .
$$

Consider a general decomposition

$$
\int d^{2} x_{1} x_{1}^{j} \nabla_{10}^{i} G_{10}^{i}(z s)=x_{10}^{j} G_{4}\left(x_{10}^{2}, z s\right)+\epsilon^{j k} x_{10}^{k} G_{5}\left(x_{10}^{2}, z s\right) .
$$

Note that the $x_{1}$ integration should be understood as keeping $\underline{x}_{10}$ fixed, that is, $\underline{x}_{0}=$ $\underline{x}_{1}-\underline{x}_{10}$. Since $G_{10}^{i}$ contains exactly one $\epsilon^{i j}$ (see its evolution equations (3.33) below along with the initial conditions (3.23) or (D.1)), we conclude that $G_{4}=0$ in the DLA and, therefore,

$$
\int d^{2} x_{1} x_{1}^{j} \nabla_{10}^{i} G_{10}^{i}(z s)=\epsilon^{j k} x_{10}^{k} G_{5}\left(x_{10}^{2}, z s\right)
$$

or, equivalently,

$$
G_{5}\left(x_{10}^{2}, z s\right)=\frac{\epsilon^{j k} x_{10}^{k}}{x_{10}^{2}} \int d^{2} x_{1} x_{1}^{j} \nabla_{10}^{i} G_{10}^{i}(z s) .
$$

The gluon OAM becomes

$$
L_{G}\left(x, Q^{2}\right)=-\frac{8 i N_{c}}{g^{2}(2 \pi)^{3}} \int d^{2} x_{10} d^{2} k_{\perp} e^{i \underline{k} \cdot \underline{x}_{10}}\left(\underline{k} \cdot \underline{x}_{10}\right) G_{5}\left(x_{10}^{2}, z s=\frac{Q^{2}}{x}\right) .
$$

For comparison, the dipole gluon helicity TMD is (see eq. (3.18))

$$
g_{1 L}^{G \operatorname{dip}}\left(x, k_{T}^{2}\right)=\frac{8 i N_{c}}{g^{2}(2 \pi)^{3}} \int d^{2} x_{10} e^{i \underline{k} \cdot \underline{x}_{10}}\left(\underline{k} \cdot \underline{x}_{10}\right) G_{2}\left(x_{10}^{2}, z s=\frac{Q^{2}}{x}\right),
$$

where

$$
\int d^{2} x_{1} G_{10}^{i}=\epsilon^{i j} x_{10}^{j} G_{2}\left(x_{10}^{2}\right)
$$


such that

$$
G_{2}\left(x_{10}^{2}\right)=\frac{\epsilon^{j k} x_{10}^{k}}{x_{10}^{2}} \int d^{2} x_{1} G_{10}^{j} .
$$

Similar to the quark helicity case, while the evolution equations for $G_{10}^{i}(z s)$ were constructed in [5], their solution was found only for the impact parameter-integrated quantity $G_{2}$. Hence no solution for $G_{10}^{i}(z s)$ exists which would allow us to simply use eq. (3.28) to find $G_{5}$. Instead we need a relation between $G_{5}$ and $G_{2}$. To obtain it we need to construct a DLA evolution equation for $G_{5}\left(x_{10}^{2}, z s\right)$ first. Our next step is to use the evolution equations for $G_{10}^{i}(z s)$ derived in [5] to obtain the evolution equations for $G_{5}\left(x_{10}^{2}, z s\right)$ using eq. (3.28). Note that, as pointed out above, at Born level $L_{G}=-\Delta G$, and, hence, $G_{5}^{(0)}=G_{2}^{(0)}$, which can also be verified independently by an explicit calculation.

\subsection{Evolution equations for gluon OAM and their solution}

Start with eqs. (96) of [5],

$$
\begin{aligned}
& G_{10}^{i}(z s)=G_{10}^{i(0)}(z s)+\frac{\alpha_{s} N_{c}}{2 \pi^{2}} \int_{\frac{\Lambda^{2}}{s}}^{z} \frac{d z^{\prime}}{z^{\prime}} \int d^{2} x_{2} \ln \frac{1}{x_{21}^{2} \Lambda^{2}} \frac{\epsilon^{i j} x_{21}^{j}}{x_{21}^{2}}\left[\Gamma_{20,21}^{\mathrm{gen}}\left(z^{\prime} s\right)+G_{21}\left(z^{\prime} s\right)\right] \\
& -\frac{\alpha_{s} N_{c}}{2 \pi^{2}} \int_{\frac{\Lambda^{2}}{s}}^{z} \frac{d z^{\prime}}{z^{\prime}} \int d^{2} x_{2} \ln \frac{1}{x_{21}^{2} \Lambda^{2}} \frac{\epsilon^{i j} x_{20}^{j}}{x_{20}^{2}}\left[\Gamma_{20,21}^{\mathrm{gen}}\left(z^{\prime} s\right)+\Gamma_{21,20}^{\mathrm{gen}}\left(z^{\prime} s\right)\right] \\
& +\frac{\alpha_{s} N_{c}}{2 \pi^{2}} \int_{\frac{1}{x_{10}^{2}}}^{z} \frac{d z^{\prime}}{z^{\prime}} \int \frac{d^{2} x_{2}}{x_{21}^{2}} \theta\left(x_{10}^{2}-x_{21}^{2}\right) \theta\left(x_{21}^{2}-\frac{1}{z^{\prime} s}\right)\left[G_{12}^{i}\left(z^{\prime} s\right)-\Gamma_{10,21}^{i}\left(z^{\prime} s\right)\right], \\
& \Gamma_{10,21}^{i}\left(z^{\prime} s\right)=G_{10}^{i(0)}\left(z^{\prime} s\right)+\frac{\alpha_{s} N_{c}}{2 \pi^{2}} \int_{\frac{\Lambda^{2}}{s}}^{z^{\prime}} \frac{d z^{\prime \prime}}{z^{\prime \prime}} \int d^{2} x_{3} \ln \frac{1}{x_{31}^{2} \Lambda^{2}} \frac{\epsilon^{i j} x_{31}^{j}}{x_{31}^{2}}\left[\Gamma_{30,31}^{\text {gen }}\left(z^{\prime \prime} s\right)+G_{31}\left(z^{\prime \prime} s\right)\right] \\
& -\frac{\alpha_{s} N_{c}}{2 \pi^{2}} \int_{\frac{\Lambda^{2}}{s}}^{z^{\prime}} \frac{d z^{\prime \prime}}{z^{\prime \prime}} \int d^{2} x_{3} \ln \frac{1}{x_{31}^{2} \Lambda^{2}} \frac{\epsilon^{i j} x_{30}^{j}}{x_{30}^{2}}\left[\Gamma_{30,31}^{\mathrm{gen}}\left(z^{\prime \prime} s\right)+\Gamma_{31,30}^{\mathrm{gen}}\left(z^{\prime \prime} s\right)\right] \\
& +\frac{\alpha_{s} N_{c}}{2 \pi^{2}} \int_{\frac{1}{x_{10}^{2}}}^{z^{\prime}} \frac{d z^{\prime \prime}}{z^{\prime \prime}} \int \frac{d^{2} x_{3}}{x_{31}^{2}} \theta\left(\min \left[x_{10}^{2}, x_{21}^{2} \frac{z^{\prime}}{z^{\prime \prime}}\right]-x_{31}^{2}\right) \theta\left(x_{31}^{2}-\frac{1}{z^{\prime \prime} s}\right)\left[G_{13}^{i}\left(z^{\prime \prime} s\right)-\Gamma_{10,31}^{i}\left(z^{\prime \prime} s\right)\right],
\end{aligned}
$$

with

$$
\Gamma_{20,21}^{\mathrm{gen}}\left(z^{\prime} s\right)=\theta\left(x_{20}-x_{21}\right) \Gamma_{20,21}\left(z^{\prime} s\right)+\theta\left(x_{21}-x_{20}\right) G_{20}\left(z^{\prime} s\right) .
$$


Employing eq. (3.28) we write for the first equation

$$
\begin{aligned}
G_{5}\left(x_{10}^{2}, z s\right)= & G_{5}^{(0)}\left(x_{10}^{2}, z s\right)+\frac{\alpha_{s} N_{c}}{2 \pi^{2}} \int_{\frac{\Lambda^{2}}{s}}^{z} \frac{d z^{\prime}}{z^{\prime}} \frac{\epsilon^{k m} x_{10}^{m}}{x_{10}^{2}} \nabla_{10}^{i} \int d^{2} x_{2} d^{2} x_{1} x_{1}^{k} \ln \frac{1}{x_{21}^{2} \Lambda^{2}} \frac{\epsilon^{i j} x_{21}^{j}}{x_{21}^{2}}\left[\Gamma_{20,21}^{\mathrm{gen}}\left(z^{\prime} s\right)+G_{21}\left(z^{\prime} s\right)\right] \\
& -\frac{\alpha_{s} N_{c}}{2 \pi^{2}} \int_{\frac{\Lambda^{2}}{s}}^{z} \frac{d z^{\prime}}{z^{\prime}} \frac{\epsilon^{k m} x_{10}^{m}}{x_{10}^{2}} \nabla_{10}^{i} \int d^{2} x_{2} d^{2} x_{1} x_{1}^{k} \ln \frac{1}{x_{21}^{2} \Lambda^{2}} \frac{\epsilon^{i j} x_{20}^{j}}{x_{20}^{2}}\left[\Gamma_{20,21}^{\text {gen }}\left(z^{\prime} s\right)+\Gamma_{21,20}^{\text {gen }}\left(z^{\prime} s\right)\right] \\
& +\frac{\alpha_{s} N_{c}}{2 \pi^{2}} \int_{\frac{1}{x_{10}}}^{z} \frac{d z^{\prime}}{z^{\prime}} \frac{\epsilon^{k m} x_{10}^{m}}{x_{10}^{2}} \nabla_{10}^{i} \int \frac{d^{2} x_{2}}{x_{21}^{2}} \theta\left(x_{10}^{2}-x_{21}^{2}\right) \theta\left(x_{21}^{2}-\frac{1}{z^{\prime} s}\right) d^{2} x_{1} x_{1}^{k}\left[G_{12}^{i}\left(z^{\prime} s\right)-\Gamma_{10,21}^{i}\left(z^{\prime} s\right)\right] .
\end{aligned}
$$

We stress that the $x_{1}$ integration should be understood as keeping $\underline{x}_{10}$ fixed. In the last term in eq. (3.35) we replace $d^{2} x_{2} \rightarrow d^{2} x_{21}$. We also note that the operator $\nabla_{10}^{i}$ should not act on the first $\theta$-function, since this would lead to a non-DLA term. We thus arrive at

$$
\begin{aligned}
G_{5}\left(x_{10}^{2}, z s\right)= & G_{5}^{(0)}\left(x_{10}^{2}, z s\right)+\frac{\alpha_{s} N_{c}}{2 \pi^{2}} \int_{\frac{\Lambda^{2}}{s}}^{z} \frac{d z^{\prime}}{z^{\prime}} \frac{\epsilon^{k m} x_{10}^{m}}{x_{10}^{2}} \nabla_{10}^{i} \int d^{2} x_{2} d^{2} x_{1} x_{1}^{k} \ln \frac{1}{x_{21}^{2} \Lambda^{2}} \frac{\epsilon^{i j} x_{21}^{j}}{x_{21}^{2}}\left[\Gamma_{20,21}^{\mathrm{gen}}\left(z^{\prime} s\right)+G_{21}\left(z^{\prime} s\right)\right] \\
& -\frac{\alpha_{s} N_{c}}{2 \pi^{2}} \int_{\frac{\Lambda^{2}}{s}}^{z} \frac{d z^{\prime}}{z^{\prime}} \frac{\epsilon^{k m} x_{10}^{m}}{x_{10}^{2}} \nabla_{10}^{i} \int d^{2} x_{2} d^{2} x_{1} x_{1}^{k} \ln \frac{1}{x_{21}^{2} \Lambda^{2}} \frac{\epsilon^{i j} x_{20}^{j}}{x_{20}^{2}}\left[\Gamma_{20,21}^{\mathrm{gen}}\left(z^{\prime} s\right)+\Gamma_{21,20}^{\mathrm{gen}}\left(z^{\prime} s\right)\right] \\
& -\frac{\alpha_{s} N_{c}}{2 \pi} \int_{\frac{1}{x_{10}^{2}}}^{z} \frac{d z^{\prime}}{z^{\prime}} \int_{1 /\left(z^{\prime} s\right)}^{x_{10}^{2}} \frac{d x_{21}^{2}}{x_{21}^{2}} \Gamma_{5}\left(x_{10}^{2}, x_{21}^{2}, z^{\prime} s\right),
\end{aligned}
$$

where

$$
\Gamma_{5}\left(x_{10}^{2}, x_{21}^{2}, z^{\prime} s\right)=\frac{\epsilon^{k m} x_{10}^{m}}{x_{10}^{2}} \nabla_{10}^{i} \int d^{2} x_{1} x_{1}^{k} \Gamma_{10,21}^{i}\left(z^{\prime} s\right) .
$$

To simplify the remaining terms on the right of eq. (3.36) we replace $d^{2} x_{2} d^{2} x_{1} \rightarrow$ $d^{2} x_{21} d^{2} x_{2}$. In section 2.3 we have shown that

$$
\int d^{2} x_{2} x_{2}^{k} \Gamma_{20,21}^{\text {gen }}\left(z^{\prime} s\right) \sim\left(z^{\prime} s\right)^{2 \sqrt{\frac{\alpha_{s} N_{c}}{2 \pi}}}, \quad \int d^{2} x_{2} x_{2}^{k} G_{21}\left(z^{\prime} s\right) \sim\left(z^{\prime} s\right)^{2 \sqrt{\frac{\alpha_{s} N_{c}}{2 \pi}}},
$$

as follows from eqs. (2.32a), (2.40a) and (2.45). For small- $x$ asymptotics of quark OAM considered above this behavior was found to be subleading. Below we will proceed assuming that the expressions in eq. (3.38) are also subleading here, and neglect these expressions when evaluating the terms on the right of eq. (3.36) containing $\Gamma_{20,21}^{\text {gen }}, G_{21}$ and $\Gamma_{21,20}^{\text {gen }}$. This approach will be justified by the fact that the term that would be left in the end of the calculation would scale with a higher power of energy than the terms in eq. (3.38). 
Neglecting the terms in eq. (3.38) we write

$$
\begin{aligned}
& \frac{\epsilon^{k m} x_{10}^{m}}{x_{10}^{2}} \nabla_{10}^{i} \int d^{2} x_{2} d^{2} x_{1} x_{1}^{k} \ln \frac{1}{x_{21}^{2} \Lambda^{2}} \frac{\epsilon^{i j} x_{21}^{j}}{x_{21}^{2}}\left[\Gamma_{20,21}^{\mathrm{gen}}\left(z^{\prime} s\right)+G_{21}\left(z^{\prime} s\right)\right] \\
& \quad=\frac{\epsilon^{k m} x_{10}^{m}}{x_{10}^{2}} \nabla_{10}^{i} \int d^{2} x_{21} d^{2} x_{2}\left(-x_{21}^{k}\right) \ln \frac{1}{x_{21}^{2} \Lambda^{2}} \frac{\epsilon^{i j} x_{21}^{j}}{x_{21}^{2}}\left[\Gamma_{20,21}^{\mathrm{gen}}\left(z^{\prime} s\right)+G_{21}\left(z^{\prime} s\right)\right] \\
& \quad=\frac{\epsilon^{k m} x_{10}^{m}}{x_{10}^{2}} \nabla_{10}^{i} \int d^{2} x_{21}\left(-x_{21}^{k}\right) \ln \frac{1}{x_{21}^{2} \Lambda^{2}} \frac{\epsilon^{i j} x_{21}^{j}}{x_{21}^{2}}\left[\Gamma^{\mathrm{gen}}\left(x_{20}^{2}, x_{21}^{2}, z^{\prime} s\right)+G\left(x_{21}^{2}, z^{\prime} s\right)\right] \\
& \quad=\int d^{2} x_{21} \frac{\underline{x}_{10} \times \underline{x}_{21}}{x_{10}^{2}} \ln \frac{1}{x_{21}^{2} \Lambda^{2}} \frac{\epsilon^{i j} x_{21}^{j}}{x_{21}^{2}} \nabla_{10}^{i} \Gamma^{\mathrm{gen}}\left(x_{20}^{2}, x_{21}^{2}, z^{\prime} s\right) .
\end{aligned}
$$

In the last step we have noticed that the $G$-term does not depend on $x_{10}$ and thus vanishes after differentiation. Note that

$$
\Gamma^{\text {gen }}\left(x_{20}^{2}, x_{21}^{2}, z^{\prime} s\right)=\int d^{2} x_{2} \Gamma_{20,21}^{\text {gen }}\left(z^{\prime} s\right)
$$

where $x_{21}$ and $\underline{x}_{20}$ are kept fixed during the integration.

The second (after the inhomogeneous) term on the right of eq. (3.36) is proportional to

$$
\begin{aligned}
& \frac{\epsilon^{k m} x_{10}^{m}}{x_{10}^{2}} \nabla_{10}^{i} \int d^{2} x_{2} d^{2} x_{1} x_{1}^{k} \ln \frac{1}{x_{21}^{2} \Lambda^{2}} \frac{\epsilon^{i j} x_{20}^{j}}{x_{20}^{2}}\left[\Gamma_{20,21}^{\mathrm{gen}}\left(z^{\prime} s\right)+\Gamma_{21,20}^{\mathrm{gen}}\left(z^{\prime} s\right)\right] \\
& \quad=\frac{\epsilon^{k m} x_{10}^{m}}{x_{10}^{2}} \nabla_{10}^{i} \int d^{2} x_{21} d^{2} x_{2}\left(-x_{21}^{k}\right) \ln \frac{1}{x_{21}^{2} \Lambda^{2}} \frac{\epsilon^{i j} x_{20}^{j}}{x_{20}^{2}}\left[\Gamma_{20,21}^{\mathrm{gen}}\left(z^{\prime} s\right)+\Gamma_{21,20}^{\mathrm{gen}}\left(z^{\prime} s\right)\right] \\
& \quad=\frac{\epsilon^{k m} x_{10}^{m}}{x_{10}^{2}} \int d^{2} x_{21}\left(-x_{21}^{k}\right) \ln \frac{1}{x_{21}^{2} \Lambda^{2}} \frac{\epsilon^{i j} x_{20}^{j}}{x_{20}^{2}} \nabla_{10}^{i}\left[\Gamma^{\mathrm{gen}}\left(x_{20}^{2}, x_{21}^{2}, z^{\prime} s\right)+\Gamma^{\mathrm{gen}}\left(x_{21}^{2}, x_{20}^{2}, z^{\prime} s\right)\right]=0
\end{aligned}
$$

since

$$
\epsilon^{i j} x_{20}^{j} \nabla_{10}^{i}\left[\Gamma^{\mathrm{gen}}\left(x_{20}^{2}, x_{21}^{2}, z^{\prime} s\right)+\Gamma^{\mathrm{gen}}\left(x_{21}^{2}, x_{20}^{2}, z^{\prime} s\right)\right] \sim \epsilon^{i j} x_{20}^{j} x_{20}^{i}=0 .
$$

We again discarded the terms in eq. (3.38) as subleading.

Substituting eqs. (3.39) and (3.41) into eq. (3.36) we arrive at

$$
\begin{aligned}
G_{5}\left(x_{10}^{2}, z s\right)= & G_{5}^{(0)}\left(x_{10}^{2}, z s\right)+\frac{\alpha_{s} N_{c}}{2 \pi^{2}} \int_{\frac{\Lambda^{2}}{s}}^{z} \frac{d z^{\prime}}{z^{\prime}} \int d^{2} x_{21} \frac{\underline{x}_{10} \times \underline{x}_{21}}{x_{10}^{2}} \ln \frac{1}{x_{21}^{2} \Lambda^{2}} \frac{\epsilon^{i j} x_{21}^{j}}{x_{21}^{2}} \nabla_{10}^{i} \Gamma^{\mathrm{gen}}\left(x_{20}^{2}, x_{21}^{2}, z^{\prime} s\right) \\
& -\frac{\alpha_{s} N_{c}}{2 \pi} \int_{\frac{1}{x_{10}^{2}}}^{z} \frac{d z^{\prime}}{z^{\prime}} \int_{1 /\left(z^{\prime} s\right)}^{x_{10}^{2}} \frac{d x_{21}^{2}}{x_{21}^{2}} \Gamma_{5}\left(x_{10}^{2}, x_{21}^{2}, z^{\prime} s\right),
\end{aligned}
$$

which is an equation containing two unknowns $\left(G_{5}, \Gamma_{5}\right)$ and a known function $\Gamma^{\mathrm{gen}}\left(x_{20}^{2}, x_{21}^{2}\right.$, $\left.z^{\prime} s\right)$. This is also similar to the gluon helicity evolution case [5]. Again the $\Gamma^{\text {gen }}\left(x_{20}^{2}, x_{21}^{2}, z^{\prime} s\right)$ 
term contains an extra $\ln s$ in the initial conditions, which makes up for the leadinglogarithmic (and not DLA) structure of the kernel acting on it in eq. (3.43) by providing one missing logarithm of energy.

Note that $[2,4]$

$$
\Gamma^{\text {gen }}\left(x_{20}^{2}, x_{21}^{2}, z^{\prime} s\right) \sim\left(z^{\prime} s\right)^{\frac{4}{\sqrt{3}}} \sqrt{\frac{\alpha_{s} N_{c}}{2 \pi}}
$$

and is dominant at high energy compared to the terms in eq. (3.38), justifying us neglecting the latter.

A set of steps similar to those needed to arrive at eq. (3.43) when applied to eq. (3.33b) gives

$$
\begin{aligned}
\Gamma_{5}\left(x_{10}^{2}, x_{21}^{2}, z^{\prime} s\right)= & G_{5}^{(0)}\left(x_{10}^{2}, z s\right)+\frac{\alpha_{s} N_{c}}{2 \pi^{2}} \int_{\frac{\Lambda^{2}}{s}}^{z^{\prime}} \frac{d z^{\prime \prime}}{z^{\prime \prime}} \int d^{2} x_{31} \frac{x_{10} \times \underline{x}_{31}}{x_{10}^{2}} \ln \frac{1}{x_{31}^{2} \Lambda^{2}} \frac{\epsilon^{i j} x_{31}^{j}}{x_{31}^{2}} \nabla_{10}^{i} \Gamma^{\mathrm{gen}}\left(x_{30}^{2}, x_{31}^{2}, z^{\prime \prime} s\right) \\
& -\frac{\alpha_{s} N_{c}}{2 \pi} \int_{\frac{1}{x_{10}}}^{z^{\prime}} \frac{d z^{\prime \prime}}{z^{\prime \prime}} \int_{1 /\left(z^{\prime \prime} s\right)}^{\min \left[x_{10}^{2}, x_{21}^{2} \frac{z^{\prime}}{z^{\prime \prime}}\right]} \frac{d x_{31}^{2}}{x_{31}^{2}} \Gamma_{5}\left(x_{10}^{2}, x_{31}^{2}, z^{\prime \prime} s\right) .
\end{aligned}
$$

We thus have the following coupled system of equations:

$$
\begin{aligned}
& G_{5}\left(x_{10}^{2}, z s\right)=G_{5}^{(0)}\left(x_{10}^{2}, z s\right)+\frac{\alpha_{s} N_{c}}{2 \pi^{2}} \int_{\frac{\Lambda^{2}}{s}}^{z} \frac{d z^{\prime}}{z^{\prime}} \int d^{2} x_{21} \frac{\underline{x}_{10} \times \underline{x}_{21}}{x_{10}^{2}} \ln \frac{1}{x_{21}^{2} \Lambda^{2}} \frac{\epsilon^{i j} x_{21}^{j}}{x_{21}^{2}} \nabla_{10}^{i} \Gamma^{\mathrm{gen}}\left(x_{20}^{2}, x_{21}^{2}, z^{\prime} s\right) \\
& -\frac{\alpha_{s} N_{c}}{2 \pi} \int_{\frac{1}{x_{10}^{2}}}^{z} \frac{d z^{\prime}}{z^{\prime}} \int_{1 /\left(z^{\prime} s\right)}^{x_{10}^{2}} \frac{d x_{21}^{2}}{x_{21}^{2}} \Gamma_{5}\left(x_{10}^{2}, x_{21}^{2}, z^{\prime} s\right) \\
& \Gamma_{5}\left(x_{10}^{2}, x_{21}^{2}, z^{\prime} s\right)=G_{5}^{(0)}\left(x_{10}^{2}, z s\right)+\frac{\alpha_{s} N_{c}}{2 \pi^{2}} \int_{\frac{\Lambda^{2}}{s}}^{z^{\prime}} \frac{d z^{\prime \prime}}{z^{\prime \prime}} \int d^{2} x_{31} \frac{x_{10} \times x_{31}}{x_{10}^{2}} \ln \frac{1}{x_{31}^{2} \Lambda^{2}} \frac{\epsilon^{i j} x_{31}^{j}}{x_{31}^{2}} \nabla_{10}^{i} \Gamma^{\operatorname{gen}}\left(x_{30}^{2}, x_{31}^{2}, z^{\prime \prime} s\right) \\
& -\frac{\alpha_{s} N_{c}}{2 \pi} \int_{\frac{1}{x_{10}^{2}}}^{z^{\prime}} \frac{d z^{\prime \prime}}{z^{\prime \prime}} \int_{1 /\left(z^{\prime \prime} s\right)}^{\min \left[x_{10}^{2}, x_{21}^{2} \frac{z^{\prime}}{z^{\prime \prime}}\right]} \frac{d x_{31}^{2}}{x_{31}^{2}} \Gamma_{5}\left(x_{10}^{2}, x_{31}^{2}, z^{\prime \prime} s\right)
\end{aligned}
$$

Equations (3.46) have the same structure as the equations for $G_{2}$ and $\Gamma_{2}$, see eqs. (98) in $[5]$,

$$
G_{2}\left(x_{10}^{2}, z s\right)=-\left(\frac{\alpha_{s} N_{c}}{3 \pi} \frac{1}{\alpha_{h}^{q}} G_{0}\right)\left(z s x_{10}^{2}\right)^{\alpha_{h}^{q}} \ln \frac{1}{x_{10}^{2} \Lambda^{2}}-\frac{\alpha_{s} N_{c}}{2 \pi} \int_{\frac{1}{x_{10}^{2}}}^{z} \frac{d z^{\prime}}{z^{\prime}} \int_{\frac{1}{z^{\prime} s}}^{x_{10}^{2}} \frac{d x_{21}^{2}}{x_{21}^{2}} \Gamma_{2}\left(x_{10}^{2}, x_{21}^{2}, z^{\prime} s\right),
$$




$$
\begin{aligned}
\Gamma_{2}\left(x_{10}^{2}, x_{21}^{2}, z^{\prime} s\right)=-\left(\frac{\alpha_{s} N_{c}}{3 \pi} \frac{1}{\alpha_{h}^{q}} G_{0}\right) & \left(z^{\prime} s x_{10}^{2}\right)^{\alpha_{h}^{q}} \ln \frac{1}{x_{10}^{2} \Lambda^{2}} \\
& -\frac{\alpha_{s} N_{c}}{2 \pi} \int_{\frac{1}{x_{10}^{2}}}^{z^{\prime}} \frac{d z^{\prime \prime}}{z^{\prime \prime}} \int_{\frac{1}{z^{\prime \prime} s}}^{\min \left[x_{10}^{2}, x_{21}^{2} \frac{z^{\prime}}{z^{\prime \prime}}\right]} \frac{d x_{31}^{2}}{x_{31}^{2}} \Gamma_{2}\left(x_{10}^{2}, x_{31}^{2}, z^{\prime \prime} s\right),
\end{aligned}
$$

where

$$
\Gamma_{2}\left(x_{10}^{2}, x_{21}^{2}, z^{\prime} s\right)=\frac{\epsilon^{j k} x_{10}^{k}}{x_{10}^{2}} \int d^{2} x_{1} \Gamma_{10,21}^{j}\left(z^{\prime} s\right) .
$$

In fact, the only difference between (3.46) and (3.47) is due to the inhomogeneous terms. In order to compare the two sets of equations, we have to compare their inhomogeneous terms. To do this, we can employ the exact solution of the impact parameterintegrated quark helicity equations (2.33) found in [4],

$$
\begin{aligned}
G\left(x_{10}^{2}, z s\right) & =\frac{1}{3} G_{0}\left(z s x_{10}^{2}\right)^{\alpha_{h}^{q}} \\
\Gamma\left(x_{10}^{2}, x_{21}^{2}, z s\right) & =\frac{1}{3} G_{0}\left(z s x_{21}^{2}\right)^{\alpha_{h}^{q}}\left[4\left(\frac{x_{10}^{2}}{x_{21}^{2}}\right)^{\frac{\alpha_{h}^{q}}{4}}-3\right],
\end{aligned}
$$

where, as in the above, the "quark helicity intercept" is given by

$$
\alpha_{h}^{q}=\frac{4}{\sqrt{3}} \sqrt{\frac{\alpha_{s} N_{c}}{2 \pi}} \approx 2.31 \sqrt{\frac{\alpha_{s} N_{c}}{2 \pi}} .
$$

$G_{0}$ is the inhomogeneous term in the impact parameter-integrated version of eqs. (2.33), assumed for simplicity to be constant in [4], and

$$
\Gamma\left(x_{10}^{2}, x_{21}^{2}, z^{\prime} s\right)=\int d^{2} x_{2} \Gamma_{10,21}\left(z^{\prime} s\right) .
$$

After a straightforward differentiation we arrive at the following expression for the second inhomogeneous term in (3.46):

$$
\begin{aligned}
& \frac{\alpha_{s} N_{c}}{2 \pi^{2}} \int_{\frac{\Lambda^{2}}{s}}^{z} \frac{d z^{\prime}}{z^{\prime}} \int d^{2} x_{21} \frac{\underline{x}_{10} \times \underline{x}_{21}}{x_{10}^{2}} \ln \frac{1}{x_{21}^{2} \Lambda^{2}} \frac{\epsilon^{i j}\left(x_{21}\right)_{\perp}^{j}}{x_{21}^{2}} \nabla_{10}^{i} \Gamma^{\mathrm{gen}}\left(x_{20}^{2}, x_{21}^{2}, z^{\prime} s\right) \\
& \quad=\frac{\alpha_{s} N_{c}}{\pi^{2}} \alpha_{h}^{q} \frac{G_{0}}{3} \int_{\frac{\Lambda^{2}}{s}}^{z} \frac{d z^{\prime}}{z^{\prime}}\left(z^{\prime} s x_{10}^{2}\right)^{\alpha_{h}^{q}} K\left(\frac{1}{x_{10}^{2} \Lambda^{2}}\right),
\end{aligned}
$$

where we have defined

$$
K\left(\frac{1}{x_{10}^{2} \Lambda^{2}}\right) \equiv \int d^{2} x_{21} \frac{\left(\underline{x}_{10} \times \underline{x}_{21}\right)^{2}}{x_{10}^{2} x_{21}^{2} x_{20}^{2}} \ln \frac{1}{x_{21}^{2} \Lambda^{2}}\left[\theta\left(x_{20}-x_{21}\right)\left(\frac{x_{21}^{2}}{x_{10}^{2}}\right)^{\alpha_{h}^{q}}\left(\frac{x_{20}^{2}}{x_{21}^{2}}\right)^{\frac{\alpha_{h}^{q}}{4}}+\theta\left(x_{21}-x_{20}\right)\left(\frac{x_{20}^{2}}{x_{10}^{2}}\right)^{\alpha_{h}^{q}}\right] .
$$


The integral in eq. (3.54) is IR-divergent. If we cut it off by $1 / \Lambda$ in the IR, and put $\alpha_{h}^{q}=0$ in it (thus neglecting higher powers of $\alpha_{s}$ not enhanced by large logarithms of energy), we get

$$
K\left(\frac{1}{x_{10}^{2} \Lambda^{2}}\right) \approx \frac{\pi}{4} \ln ^{2}\left(\frac{1}{x_{10}^{2} \Lambda^{2}}\right)
$$

A more careful evaluation of eq. (3.54), neglecting $\alpha_{h}^{q}$ only compared to order-one constants, yields

$$
K\left(\frac{1}{x_{10}^{2} \Lambda^{2}}\right) \approx \frac{\pi}{2}\left(1-\ln \frac{1}{x_{10}^{2} \Lambda^{2}}\right)+\frac{\pi}{2\left(\alpha_{h}^{q}\right)^{2}}\left[\left(\frac{1}{x_{10}^{2} \Lambda^{2}}\right)^{\alpha_{h}^{q}}-1-\alpha_{h}^{q} \ln \frac{1}{x_{10}^{2} \Lambda^{2}}\right] .
$$

The evolution equations for $G_{5}$ and $\Gamma_{5}$ become

$$
\begin{aligned}
G_{5}\left(x_{10}^{2}, z s\right)= & G_{5}^{(0)}\left(x_{10}^{2}, z s\right)+\frac{\alpha_{s} N_{c}}{\pi^{2}} \frac{G_{0}}{3} K\left(\frac{1}{x_{10}^{2} \Lambda^{2}}\right)\left(z s x_{10}^{2}\right)^{\alpha_{h}^{q}}-\frac{\alpha_{s} N_{c}}{2 \pi} \int_{\frac{1}{x_{10} s}}^{z} \frac{d z^{\prime}}{z^{\prime}} \int_{1 /\left(z^{\prime} s\right)}^{x_{10}^{2}} \frac{d x_{21}^{2}}{x_{21}^{2}} \Gamma_{5}\left(x_{10}^{2}, x_{21}^{2}, z^{\prime} s\right), \\
\Gamma_{5}\left(x_{10}^{2}, x_{21}^{2}, z^{\prime} s\right)= & G_{5}^{(0)}\left(x_{10}^{2}, z^{\prime} s\right)+\frac{\alpha_{s} N_{c}}{\pi^{2}} \frac{G_{0}}{3} K\left(\frac{1}{x_{10}^{2} \Lambda^{2}}\right)\left(z^{\prime} s x_{10}^{2}\right)^{\alpha_{h}^{q}} \\
& -\frac{\alpha_{s} N_{c}}{2 \pi} \int_{\frac{1}{x_{10}^{2}}}^{z^{\prime}} \frac{d z^{\prime \prime}}{z^{\prime \prime}} \int_{1 /\left(z^{\prime \prime} s\right)}^{\min \left[x_{10}^{2}, x_{21}^{2} z_{z^{\prime \prime}}\right]} \frac{d x_{31}^{2}}{x_{31}^{2}} \Gamma_{5}\left(x_{10}^{2}, x_{31}^{2}, z^{\prime \prime} s\right) .
\end{aligned}
$$

Note that, as follows from eq. (3.23) above,

$$
G_{5}^{(0)}\left(x_{10}^{2}, z s\right)=G_{2}^{(0)}\left(x_{10}^{2}, z s\right)=-\frac{\alpha_{s}^{2} C_{F}}{N_{c}} \pi \ln \left(\frac{1}{x_{10} \Lambda}\right) .
$$

Equations (3.57) have two inhomogeneous terms. The following calculation would be simplified if we could neglect one compared to the other. To see which one to neglect, let us first do some power counting. The initial condition for the quark helicity evolution is of the order $G_{0} \sim \alpha_{s}^{2} \ln s \sim \alpha_{s}^{3 / 2}$ [5] if we assume that $\ln s \sim 1 / \sqrt{\alpha_{s}}$, as is appropriate for the DLA limit. We thus have, for the two inhomogeneous terms in eqs. (3.57),

$$
G_{5}^{(0)} \sim \alpha_{s}^{2} \gg \alpha_{s} G_{0} K \sim \alpha_{s}^{5 / 2}
$$

It appears that we can neglect the second inhomogeneous term compared to the first one. (Note that the situation here is slightly different from the equations for gluon helicity obtained in [5], where the second inhomogeneous term is, parametrically, of the same order as the first term, $\sqrt{\alpha_{s}} G_{0} \sim \alpha_{s}^{2} \sim G_{2}^{(0)}$, and the first term is neglected due to the lack of power of $z s$ enhancement at Born level.) However, since equations (3.57) are linear, their solution is a sum of solutions of the same equations with one set of equations having only the $G_{5}^{(0)}$ inhomogeneous terms, while another one containing only the $\alpha_{s} G_{0} K$ inhomogeneous terms. 
To find the solution of the former equations, keep only the $G_{5}^{(0)}$ inhomogeneous terms in eqs. (3.57) and substitute eq. (3.58) into eqs. (3.57). We arrive at

$$
\begin{gathered}
G_{5}\left(x_{10}^{2}, z s\right)=-\frac{\alpha_{s}^{2} C_{F}}{N_{c}} \pi \ln \left(\frac{1}{x_{10} \Lambda}\right)-\frac{\alpha_{s} N_{c}}{2 \pi} \int_{\frac{1}{x_{10}^{2}}}^{z} \frac{d z^{\prime}}{z^{\prime}} \int_{1 /\left(z^{\prime} s\right)}^{x_{10}^{2}} \frac{d x_{21}^{2}}{x_{21}^{2}} \Gamma_{5}\left(x_{10}^{2}, x_{21}^{2}, z^{\prime} s\right), \\
\Gamma_{5}\left(x_{10}^{2}, x_{21}^{2}, z^{\prime} s\right)=-\frac{\alpha_{s}^{2} C_{F}}{N_{c}} \pi \ln \left(\frac{1}{x_{10} \Lambda}\right)-\frac{\alpha_{s} N_{c}}{2 \pi} \int_{\frac{1}{x_{10}^{2}}}^{z^{\prime}} \frac{d z^{\prime \prime}}{z^{\prime \prime}} \int_{1 /\left(z^{\prime \prime} s\right)}^{\min \left[x_{10}^{2}, x_{21}^{2} \frac{z^{\prime}}{z^{\prime \prime}}\right]} \frac{d x_{31}^{2}}{x_{31}^{2}} \Gamma_{5}\left(x_{10}^{2}, x_{31}^{2}, z^{\prime \prime} s\right) .
\end{gathered}
$$

Defining

$$
\bar{G}_{5}\left(x_{10}^{2}, z s\right)=-\frac{G_{5}\left(x_{10}^{2}, z s\right)}{\frac{\alpha_{s}^{2} C_{F}}{N_{c}} \pi \ln \left(\frac{1}{x_{10} \Lambda}\right)}, \quad \bar{\Gamma}_{5}\left(x_{10}^{2}, x_{21}^{2}, z^{\prime} s\right)=-\frac{\Gamma_{5}\left(x_{10}^{2}, x_{21}^{2}, z^{\prime} s\right)}{\frac{\alpha_{s}^{2} C_{F}}{N_{c}} \pi \ln \left(\frac{1}{x_{10} \Lambda}\right)}
$$

we reduce eqs. (3.60) to

$$
\begin{aligned}
& \bar{G}_{5}\left(x_{10}^{2}, z s\right)=1-\frac{\alpha_{s} N_{c}}{2 \pi} \int_{\frac{1}{x_{10}^{2}}}^{z} \frac{d z^{\prime}}{z^{\prime}} \int_{1 /\left(z^{\prime} s\right)}^{x_{10}^{2}} \frac{d x_{21}^{2}}{x_{21}^{2}} \bar{\Gamma}_{5}\left(x_{10}^{2}, x_{21}^{2}, z^{\prime} s\right), \\
& \bar{\Gamma}_{5}\left(x_{10}^{2}, x_{21}^{2}, z^{\prime} s\right)=1-\frac{\alpha_{s} N_{c}}{2 \pi} \int_{\frac{1}{x_{10}^{2}}}^{z^{\prime}} \frac{d z^{\prime \prime}}{z^{\prime \prime}} \int_{1 /\left(z^{\prime \prime} s\right)}^{\min \left[x_{10}^{2}, x_{21}^{2} \frac{z^{\prime}}{z^{\prime \prime}}\right]} \frac{d x_{31}^{2}}{x_{31}^{2}} \bar{\Gamma}_{5}\left(x_{10}^{2}, x_{31}^{2}, z^{\prime \prime} s\right),
\end{aligned}
$$

which are solved in appendix B (for $\beta=-1$ there) with the solution for $\bar{G}_{5}$ given by eq. (B.16). Employing the latter we write

$$
G_{5}\left(s_{10}, \eta\right)=-\frac{\alpha_{s}^{2} C_{F}}{N_{c}} \pi \ln \left(\frac{1}{x_{10} \Lambda}\right) \frac{J_{1}\left(\sqrt{\frac{2 \alpha_{s} N_{c}}{\pi}} \ln \left(z s x_{10}^{2}\right)\right)}{\sqrt{\frac{\alpha_{s} N_{c}}{2 \pi}} \ln \left(z s x_{10}^{2}\right)} .
$$

This result also oscillates with a decreasing amplitude for increasing $z s x_{10}^{2}$. As we will shortly see, it is negligible compared to the solution of eqs. (3.57) with the $\alpha_{s} G_{0} K$ inhomogeneous terms.

Keeping only the $\alpha_{s} G_{0} K$ inhomogeneous terms in eqs. (3.57) we have

$$
\begin{aligned}
& G_{5}\left(x_{10}^{2}, z s\right)=\frac{\alpha_{s} N_{c}}{\pi^{2}} \frac{G_{0}}{3} K\left(\frac{1}{x_{10}^{2} \Lambda^{2}}\right)\left(z s x_{10}^{2}\right)^{\alpha_{h}^{q}}-\frac{\alpha_{s} N_{c}}{2 \pi} \int_{\frac{1}{x_{10}^{2}}}^{z} \frac{d z^{\prime}}{z^{\prime}} \int_{1 /\left(z^{\prime} s\right)}^{x_{10}^{2}} \frac{d x_{21}^{2}}{x_{21}^{2}} \Gamma_{5}\left(x_{10}^{2}, x_{21}^{2}, z^{\prime} s\right), \\
& \Gamma_{5}\left(x_{10}^{2}, x_{21}^{2}, z^{\prime} s\right)=\frac{\alpha_{s} N_{c}}{\pi^{2}} \frac{G_{0}}{3} K\left(\frac{1}{x_{10}^{2} \Lambda^{2}}\right)\left(z^{\prime} s x_{10}^{2}\right)^{\alpha_{h}^{q}}-\frac{\alpha_{s} N_{c}}{2 \pi} \int_{\frac{1}{x_{10}^{2}}}^{z^{\prime}} \frac{d z^{\prime \prime}}{z^{\prime \prime}} \int_{1 /\left(z^{\prime \prime} s\right)}^{\min \left[x_{10}^{2}, x_{21}^{2}{\frac{z^{\prime}}{z^{\prime \prime}}}^{\prime \prime}\right.} \frac{d x_{31}^{2}}{x_{31}^{2}} \Gamma_{5}\left(x_{10}^{2}, x_{31}^{2}, z^{\prime \prime} s\right) .
\end{aligned}
$$


Comparing (3.64) to (3.47) we conclude that

$$
G_{5}\left(x_{10}^{2}, z s\right)=-\frac{\alpha_{h}^{q} K\left(\frac{1}{x_{10}^{2} \Lambda^{2}}\right)}{\pi \ln \left(\frac{1}{x_{10}^{2} \Lambda^{2}}\right)} G_{2}\left(x_{10}^{2}, z s\right) .
$$

Since, as was shown in [5],

$$
G_{2}\left(x_{10}^{2}, z s\right) \sim(z s)^{\frac{13}{4 \sqrt{3}} \sqrt{\frac{\alpha_{s} N_{c}}{2 \pi}}}
$$

we conclude that

$$
G_{5}\left(x_{10}^{2}, z s\right) \sim(z s)^{\frac{13}{4 \sqrt{3}} \sqrt{\frac{\alpha_{s} N_{c}}{2 \pi}}},
$$

and the solution of eqs. (3.64) dominates over eq. (3.63) at high energy. Hence, while the $G_{5}^{(0)}$ inhomogeneous terms in eqs. (3.57) are parametrically larger than the $\alpha_{s} G_{0} K$ inhomogeneous terms, we are justified to only keep the latter, since at high energies they give the dominant contribution. Therefore, eq. (3.65) gives us the leading high-energy solution of eqs. (3.57) for $G_{5}$.

\subsection{Gluon OAM distribution at small $x$}

Using eq. (3.65) in eq. (3.29) we obtain

$$
L_{G}\left(x, Q^{2}\right)=\frac{8 i N_{c}}{g^{2}(2 \pi)^{3}} \int d^{2} x_{10} d^{2} k_{\perp} e^{i \underline{k} \cdot \underline{x}_{10}}\left(\underline{k} \cdot \underline{x}_{10}\right) \frac{\alpha_{h}^{q} K\left(\frac{1}{x_{10}^{2} \Lambda^{2}}\right)}{\pi \ln \left(\frac{1}{x_{10}^{2} \Lambda^{2}}\right)} G_{2}\left(x_{10}^{2}, s\right) .
$$

This has to be compared with the gluon helicity distribution (see (3.30))

$$
\Delta G\left(x, Q^{2}\right)=\frac{8 i N_{c}}{g^{2}(2 \pi)^{3}} \int d^{2} x_{10} d^{2} k_{\perp} e^{i \underline{k} \cdot \underline{x}_{10}}\left(\underline{k} \cdot \underline{x}_{10}\right) G_{2}\left(x_{10}^{2}, s\right) .
$$

Assuming that, after all integrations are carried out, at the leading DLA level we can simply replace

$$
\frac{1}{x_{10}^{2}} \rightarrow Q^{2}
$$

with $Q^{2}$ being the upper cutoff on the $k_{T}$ integral in both (3.68) and (3.69), we arrive at

$$
L_{G}\left(x, Q^{2}\right)=\frac{\alpha_{h}^{q} K\left(\frac{Q^{2}}{\Lambda^{2}}\right)}{\pi \ln \left(\frac{Q^{2}}{\Lambda^{2}}\right)} \Delta G\left(x, Q^{2}\right) .
$$

Noticing that the first term on the right-hand side of eq. (3.56) can be thought of as a constant under one of the logarithms in the $\alpha_{h}^{q}$-expansion of the second term, we keep only this second term on the right-hand side of (3.56) to write

$$
L_{G}\left(x, Q^{2}\right)=\frac{\left(\frac{Q^{2}}{\Lambda^{2}}\right)^{\alpha_{h}^{q}}-1-\alpha_{h}^{q} \ln \frac{Q^{2}}{\Lambda^{2}}}{2 \alpha_{h}^{q} \ln \left(\frac{Q^{2}}{\Lambda^{2}}\right)} \Delta G\left(x, Q^{2}\right) .
$$


Unlike the quark OAM in eq. (2.50), this result appears to be different from the DGLAPbased conclusion reached in [16]. However, the conclusion in [16] was based on the assumption that $|\Delta G| \gg|\Delta q|$, which is the opposite of what was found in the DLA approximation $[2,4,5]$.

The prefactor in the relation (3.72) resums powers of $\alpha_{s} \ln ^{2} \frac{Q^{2}}{\Lambda^{2}}$, which are strictlyspeaking are not DLA. (In the DLA approximation one only keeps powers of $\alpha_{s} \ln ^{2}(1 / x)$.) Therefore, it is possible that one has to expand eq. (3.72) to the lowest non-trivial order in $\alpha_{h}^{q}$, obtaining

$$
L_{G}\left(x, Q^{2}\right)=\left(\frac{\alpha_{h}^{q}}{4} \ln \frac{Q^{2}}{\Lambda^{2}}\right) \Delta G\left(x, Q^{2}\right)
$$

The small- $x$ asymptotics of the gluon OAM easily follows from either eq. (3.72) or eq. (3.73) using the DLA asymptotics of the gluon helicity distribution found in [5]. We conclude that

$$
L_{G}\left(x, Q^{2}\right) \sim \Delta G\left(x, Q^{2}\right) \sim\left(\frac{1}{x}\right)^{\alpha_{h}^{G}} \sim\left(\frac{1}{x}\right)^{\frac{13}{4 \sqrt{3}} \sqrt{\frac{\alpha_{s} N_{c}}{2 \pi}}} \sim\left(\frac{1}{x}\right)^{1.88 \sqrt{\frac{\alpha_{s} N_{c}}{2 \pi}}} .
$$

In appendix D we present a simple toy model describing a way of thinking about the DLA evolution for gluon OAM changing its relation to the gluon helicity from $L_{G}=-\Delta G$ to eq. (3.73).

\section{Summary}

The calculation performed in this paper heavily relied on the earlier works [1-6]. By simplifying the quark and gluon OAM distributions definitions at small $x$ to eqs. (2.27) and (3.25) we managed to relate these quantities to the polarized dipole amplitudes $G_{10}(z s)$ and $G_{10}^{i}(z s)$ respectively, which were employed previously for determination of the quark and gluon helicity distributions. The small- $x$ asymptotics of $L_{q+\bar{q}}\left(x, Q^{2}\right)$ and $L_{G}\left(x, Q^{2}\right)$ should have resulted from the high-energy asymptotics of these quantities. Naively, the latter could be easily found from the solutions of the DLA evolution equations for $G_{10}(z s)$ and $G_{10}^{i}(z s)$ constructed in $[2,4,5]$. However, one had to be careful here, since in $[2,4,5]$ we found the expressions for $G_{10}(z s)$ and $G_{10}^{i}(z s)$ integrated over all impact parameters. At the same time, eqs. (2.27) and (3.25) contain $G_{10}(z s)$ and $G_{10}^{i}(z s)$ respectively, weighted by the position of the polarized quark $\underline{x}_{1}$, and then integrated over all impact parameters. So an analysis of the first $\underline{x}_{1}$-moments of $G_{10}(z s)$ and $G_{10}^{i}(z s)$ was in order.

In the quark OAM case, the first $\underline{x}_{1}$-moment of $G_{10}(z s)$ turned out to be subleading at small $x$, such that eq. (2.27), after we discarded the first term on its right (which was proportional to the first moment), led to (cf. [16])

$$
L_{q+\bar{q}}\left(x, Q^{2}\right)=-\Delta \Sigma\left(x, Q^{2}\right) \sim\left(\frac{1}{x}\right)^{\frac{4}{\sqrt{3}} \sqrt{\frac{\alpha_{s} N_{c}}{2 \pi}}} .
$$


The gluon OAM distribution in eq. (3.25) is directly proportional to the first $\underline{x}_{1}$ moment of $G_{10}^{i}(z s)$. Constructing the small- $x$ asymptotics of this moment we arrived at

$$
L_{G}\left(x, Q^{2}\right)=\left(\frac{\alpha_{h}^{q}}{4} \ln \frac{Q^{2}}{\Lambda^{2}}\right) \Delta G\left(x, Q^{2}\right) \sim\left(\frac{1}{x}\right)^{\frac{13}{4 \sqrt{3}} \sqrt{\frac{\alpha_{S} N_{c}}{2 \pi}}} .
$$

Equations (4.1) and (4.2) summarize our main results in this paper.

One may be concerned about an apparent asymmetry: the first $\underline{x}_{1}$-moment of $G_{10}(z s)$ is subleading at small $x$, while the first $\underline{x}_{1}$-moment of $G_{10}^{i}(z s)$, labeled $G_{5}$ above, appears not to be subleading, and leads to eq. (4.2). Note, however, that in the strict DLA analysis the prefactor on the right of eq. (4.2) is subleading, $\sim \alpha_{h}^{q} \ln \left(Q^{2} / \Lambda^{2}\right) \sim \sqrt{\alpha_{s}} \ln \left(Q^{2} / \Lambda^{2}\right) \ll$ 1. Therefore, the first moment $G_{5}$, and, consequently, $L_{G}\left(x, Q^{2}\right)$ at small $x$ are also subleading, by apparent analogy to the quark OAM case. This is probably the consequence of keeping only the parametrically subleading but dominant at high energy inhomogeneous term $\alpha_{s} G_{0} K$ in eqs. (3.57) to arrive at the solution (3.65) for $G_{5}$. The difference in the gluon OAM case as compared to the quark OAM is that, while subleading in DLA, the $G_{5}$ term gives us the only contribution to $L_{G}\left(x, Q^{2}\right)$ and cannot be neglected. One may even speculate that our conclusion (4.2) could be more conservatively formulated as $\left|L_{G}\right| \ll|\Delta G|$ at small $x$.

\section{Acknowledgments}

Special thanks go to Feng Yuan for suggesting that the author look at quark and gluon OAM at small $x$ and to Yoshitaka Hatta for many critical discussions of this work. The author would also like to thank Matt Sievert for his interest in this project.

This material is based upon work supported by the U.S. Department of Energy, Office of Science, Office of Nuclear Physics under Award Number DE-SC0004286. The author thanks the U.S. Department of Energy's Institute for Nuclear Theory at the University of Washington for its hospitality and support during the final stages of this work (INT pre-print number INT-PUB-18-065).

\section{A The saturation/CGC averaging}

Start with the an expectation value of some operator $\hat{\mathcal{O}}(b, r)$ in the proton state $|P\rangle$. The expectation value $\langle P|\hat{\mathcal{O}}(b, r)| P\rangle$ is independent of $b=\left(b^{-}, \underline{b}\right)$. It should be proportional to the CGC-averaged operator, integrated over all space:

$$
\langle P|\hat{\mathcal{O}}(b, r)| P\rangle \sim \int d^{2} b d b^{-}\langle\hat{\mathcal{O}}(b, r)\rangle .
$$

To fix the normalization we put $\hat{\mathcal{O}}(b, r)=1$ and note that $\langle P \mid P\rangle=2 P^{+}(2 \pi)^{3} \delta\left(0^{-}\right) \delta^{2}(\underline{0})=$ $2 P^{+} V^{-}$with $V^{-}=\int d^{2} x d x^{-}$. We get

$$
\langle P|\hat{\mathcal{O}}(b, r)| P\rangle=2 P^{+} \int d^{2} b d b^{-}\langle\hat{\mathcal{O}}(b, r)\rangle .
$$


Now, since $\langle P|\hat{\mathcal{O}}(b, r)| P\rangle=\langle P|\hat{\mathcal{O}}(0, r)| P\rangle$, the off-forward matrix element is a Fourier transform of the CGC-averaged operator, with the normalization fixed by eq. (A.2),

$$
\left\langle P+\frac{\Delta}{2}|\hat{\mathcal{O}}(0, r)| P-\frac{\Delta}{2}\right\rangle=2 P^{+} \int d^{2} b d b^{-} e^{-i b \cdot \Delta}\langle\hat{\mathcal{O}}(b, r)\rangle,
$$

where $\Delta=\left(\Delta^{+}, \underline{\Delta}\right)$. (The sign in the Fourier exponent is due to $i(0-b) \cdot \Delta=-i b \cdot \Delta$.) Inverting this Fourier transform we arrive at

$$
\langle\hat{\mathcal{O}}(b, r)\rangle=\frac{1}{2 P^{+}} \int \frac{d^{2} \Delta d \Delta^{+}}{(2 \pi)^{3}} e^{i b \cdot \Delta}\left\langle P+\frac{\Delta}{2}|\hat{\mathcal{O}}(0, r)| P-\frac{\Delta}{2}\right\rangle .
$$

\section{B Solution of a useful system of integral equations}

In the main text, on two separate occasions, we needed to solve the following system of integral questions

$$
\begin{gathered}
\bar{G}_{5}\left(x_{10}^{2}, z s\right)=1+\beta \frac{\alpha_{s} N_{c}}{2 \pi} \int_{\frac{1}{x_{10}^{2}}}^{z} \frac{d z^{\prime}}{z^{\prime}} \int_{1 /\left(z^{\prime} s\right)}^{x_{10}^{2}} \frac{d x_{21}^{2}}{x_{21}^{2}} \bar{\Gamma}_{5}\left(x_{10}^{2}, x_{21}^{2}, z^{\prime} s\right), \\
\bar{\Gamma}_{5}\left(x_{10}^{2}, x_{21}^{2}, z^{\prime} s\right)=1+\beta \frac{\alpha_{s} N_{c}}{2 \pi} \int_{\frac{1}{x_{10}^{2}}}^{z^{\prime}} \frac{d z^{\prime \prime}}{z^{\prime \prime}} \int_{1 /\left(z^{\prime \prime} s\right)}^{\min \left[x_{10}^{2}, x_{21}^{2} \frac{z^{\prime}}{z^{\prime \prime}}\right]} \frac{d x_{31}^{2}}{x_{31}^{2}} \bar{\Gamma}_{5}\left(x_{10}^{2}, x_{31}^{2}, z^{\prime \prime} s\right),
\end{gathered}
$$

with eqs. (2.43) corresponding to the $\beta=+1$ case and eqs. (3.62) corresponding to the $\beta=-1$ case.

Introducing the scaled logarithmic variables [2]

$$
\begin{aligned}
\eta & \equiv \sqrt{\frac{\alpha_{s} N_{c}}{2 \pi}} \ln \frac{z s}{\Lambda^{2}}, & s_{10} & \equiv \sqrt{\frac{\alpha_{s} N_{c}}{2 \pi}} \ln \frac{1}{x_{10}^{2} \Lambda^{2}}, \\
\eta^{\prime} & \equiv \sqrt{\frac{\alpha_{s} N_{c}}{2 \pi}} \ln \frac{z^{\prime} s}{\Lambda^{2}}, & s_{21} & \equiv \sqrt{\frac{\alpha_{s} N_{c}}{2 \pi}} \ln \frac{1}{x_{21}^{2} \Lambda^{2}}, \\
\eta^{\prime \prime} & \equiv \sqrt{\frac{\alpha_{s} N_{c}}{2 \pi}} \ln \frac{z^{\prime \prime} s}{\Lambda^{2}}, & s_{32} & \equiv \sqrt{\frac{\alpha_{s} N_{c}}{2 \pi}} \ln \frac{1}{x_{32}^{2} \Lambda^{2}}
\end{aligned}
$$

we get

$$
\begin{gathered}
\bar{G}_{5}\left(s_{10}, \eta\right)=1+\beta \int_{s_{10}}^{\eta} d \eta^{\prime} \int_{s_{10}}^{\eta^{\prime}} d s_{21} \bar{\Gamma}_{5}\left(s_{10}, s_{21}, \eta^{\prime}\right), \\
\bar{\Gamma}_{5}\left(s_{10}, s_{21}, \eta^{\prime}\right)=1+\beta \int_{s_{10}}^{\eta^{\prime}} d \eta^{\prime \prime} \int_{\max \left\{s_{10}, s_{21}+\eta^{\prime \prime}-\eta^{\prime}\right\}}^{\eta^{\prime \prime}} d s_{32} \bar{\Gamma}_{5}\left(s_{10}, s_{32}, \eta^{\prime \prime}\right) .
\end{gathered}
$$


By analogy to [4] we assume a scaling ansatz of the solution,

$$
\begin{aligned}
\bar{G}_{5}\left(s_{10}, \eta\right) & =\bar{G}_{5}\left(\eta-s_{10}\right), \\
\bar{\Gamma}_{5}\left(s_{10}, s_{21}, \eta^{\prime}\right) & =\bar{\Gamma}_{5}\left(\eta^{\prime}-s_{10}, \eta^{\prime}-s_{21}\right) .
\end{aligned}
$$

Equations (B.3) become

$$
\begin{aligned}
\bar{G}_{5}(\zeta) & =1+\beta \int_{0}^{\zeta} d \xi \int_{0}^{\xi} d \xi^{\prime} \bar{\Gamma}_{5}\left(\xi, \xi^{\prime}\right), \\
\bar{\Gamma}_{5}\left(\zeta, \zeta^{\prime}\right) & =1+\beta \int_{0}^{\zeta^{\prime}} d \xi \int_{0}^{\xi} d \xi^{\prime} \bar{\Gamma}_{5}\left(\xi, \xi^{\prime}\right)+\beta \int_{\zeta^{\prime}}^{\zeta} d \xi \int_{0}^{\zeta^{\prime}} d \xi^{\prime} \bar{\Gamma}_{5}\left(\xi, \xi^{\prime}\right),
\end{aligned}
$$

Following [4] we write eqs. (B.6) in a differential form,

$$
\begin{aligned}
\partial_{\zeta} \bar{G}_{5}(\zeta) & =\beta \int_{0}^{\zeta} d \xi^{\prime} \bar{\Gamma}_{5}\left(\zeta, \xi^{\prime}\right), \\
\partial_{\zeta} \bar{\Gamma}_{5}\left(\zeta, \zeta^{\prime}\right) & =\beta \int_{0}^{\zeta^{\prime}} d \xi^{\prime} \bar{\Gamma}_{5}\left(\zeta, \xi^{\prime}\right),
\end{aligned}
$$

with the initial conditions

$$
\bar{G}_{5}(0)=1, \quad \bar{\Gamma}_{5}(\xi, \xi)=\bar{G}_{5}(\xi) .
$$

These equations can be solved with the help of a Laplace transform, leading to

$$
\begin{aligned}
\bar{\Gamma}_{5}\left(\zeta, \zeta^{\prime}\right) & =\int \frac{d \omega}{2 \pi i} e^{\omega \zeta^{\prime}+\frac{\beta \zeta}{\omega}} \Gamma_{5 \omega}(0), \\
\bar{G}_{5}(\zeta) & =\bar{\Gamma}_{5}(\zeta, \zeta)=\int \frac{d \omega}{2 \pi i} e^{\left(\omega+\frac{\beta}{\omega}\right) \zeta} \Gamma_{5 \omega}(0)
\end{aligned}
$$

with the still unknown function $\Gamma_{5 \omega}(0)$ satisfying the following relations:

$$
\int \frac{d \omega}{2 \pi i} e^{\frac{\beta \zeta}{\omega}} \frac{1}{\omega} \Gamma_{5 \omega}(0)=0, \quad \quad \int \frac{d \omega}{2 \pi i} e^{\left(\omega+\frac{\beta}{\omega}\right) \zeta} \omega \Gamma_{5 \omega}(0)=0 .
$$

Searching for $\Gamma_{5 \omega}(0)$ in the form

$$
\Gamma_{5 \omega}(0)=\sum_{n=-\infty}^{n=\infty} c_{n} \omega^{n}
$$

and satisfying the constraints (B.10) we arrive at

$$
\Gamma_{5 \omega}(0)=c_{-1}\left[\frac{1}{\omega}-\frac{\beta}{\omega^{3}}\right] .
$$


Inserting eq. (B.12) into eqs. (B.9) yields

$$
\begin{aligned}
\bar{G}_{5}(\zeta) & =\int \frac{d \omega}{2 \pi i} e^{\left(\omega+\frac{\beta}{\omega}\right) \zeta}\left[\frac{1}{\omega}-\frac{\beta}{\omega^{3}}\right], \\
\bar{\Gamma}_{5}\left(\zeta, \zeta^{\prime}\right) & =\int \frac{d \omega}{2 \pi i} e^{\omega \zeta^{\prime}+\frac{\beta \zeta}{\omega}}\left[\frac{1}{\omega}-\frac{\beta}{\omega^{3}}\right],
\end{aligned}
$$

where we have fixed $c_{-1}=1$ by imposing the $\bar{G}_{5}(0)=1$ condition.

Since we are interested in $\bar{G}_{5}(\zeta)$, we perform the $\omega$-integral in eq. (B.13a), arriving at

$$
\bar{G}_{5}(\zeta)=\frac{I_{1}(2 \zeta \sqrt{\beta})}{\zeta \sqrt{\beta}}
$$

For $\beta=+1$, eq. (B.14) yields

$$
\bar{I}\left(s_{10}, \eta\right)=\bar{G}_{5}\left(s_{10}, \eta\right)=\frac{I_{1}\left(2\left(\eta-s_{10}\right)\right)}{\eta-s_{10}}=\frac{I_{1}\left(2 \sqrt{\frac{\alpha_{s} N_{c}}{2 \pi}} \ln \left(z s x_{10}^{2}\right)\right)}{\sqrt{\frac{\alpha_{s} N_{c}}{2 \pi}} \ln \left(z s x_{10}^{2}\right)} .
$$

For $\beta=-1$, eq. (B.14) gives

$$
\bar{G}_{5}\left(s_{10}, \eta\right)=\frac{J_{1}\left(2\left(\eta-s_{10}\right)\right)}{\eta-s_{10}}=\frac{J_{1}\left(\sqrt{\frac{2 \alpha_{s} N_{c}}{\pi}} \ln \left(z s x_{10}^{2}\right)\right)}{\sqrt{\frac{\alpha_{s} N_{c}}{2 \pi}} \ln \left(z s x_{10}^{2}\right)} .
$$

\section{Comparison with the earlier works}

Here we demonstrate that our definition (3.4) of the gluon OAM agrees with that in $[7,18]$. Therefore, we are using the Jaffe-Manohar definition of the gluon OAM. Our strategy is to show that the gluon OAM definitions in $[7,18]$ are equivalent to each other, and that our definition is equivalent to [18], and, hence, to [7].

Begin with eq. (4) in [18], which we can write as follows:

$$
\begin{aligned}
L_{G} & =-\frac{i \epsilon^{i j}}{2 S^{+}} \lim _{\Delta \rightarrow 0} \frac{\delta}{\delta \Delta^{j}}\left\langle P^{\prime}, S\left|F^{+\alpha}(0) \overleftrightarrow{D}_{\text {pure }}^{i} A_{\alpha}^{\text {phys }}(0)\right| P, S\right\rangle \\
& =\frac{\epsilon^{i j}}{2 S^{+} V^{-}} \int d^{2} x d x^{-} x^{j}\left\langle P, S\left|F^{+\alpha}(x) \overleftrightarrow{D}_{\text {pure }}^{i} A_{\alpha}^{\text {phys }}(x)\right| P, S\right\rangle,
\end{aligned}
$$

where $V^{-}=\int d^{2} x d x^{-}=(2 \pi)^{3} \delta^{2}(\underline{0}) \delta\left(0^{+}\right)$and $\Delta^{\mu}=P^{\prime \mu}-P^{\mu}$.

For simplicity, let us work in the $A^{+}=0$ gauge with the $\underline{\nabla} \cdot \underline{A}\left(x^{-}=-\infty\right)=0$ subgauge condition [54]. Then $A_{\alpha}^{\text {phys }}(x)=A_{\alpha}(x)$. Eq. (C.1) becomes (after integration by parts)

$$
L_{G}=\frac{1}{2 S^{+} V^{-}} \int d^{2} x d x^{-}\left\langle P, S\left|F^{+\alpha}(x)(\underline{x} \times \underline{\nabla}) A_{\alpha}(x)\right| P, S\right\rangle .
$$


Assuming that color trace (2 tr) is implied in eq. (4) of [18], and using $S^{+}=P^{+}$we write eq. (C.2) as

$$
L_{G}=-\frac{1}{2 P^{+} V^{-}} \int d^{2} x d x^{-}\left\langle P, S\left|2 \operatorname{tr}\left[F^{+i}(x)(\underline{x} \times \underline{\nabla}) A^{i}(x)\right]\right| P, S\right\rangle .
$$

Observing that $E^{i}=-F^{0 i}=-\left(F^{+i}+F^{-i}\right) / \sqrt{2}$ and neglecting $F^{-i}$ in the infinite momentum frame (which we can do even for the sub-eikonal helicity-dependent gluon field, as it appears that helicity-dependent part of $F^{-i}$ is sub-sub-eikonal) we get $F^{+i}=-\sqrt{2} E^{i}$. On the other hand, $P^{+}=\sqrt{2} E$, with $E$ the energy of the proton. We get

$$
L_{G}=\frac{1}{2 E V^{-}} \int d^{2} x d x^{-}\left\langle P, S\left|2 \operatorname{tr}\left[E^{i}(x)(\underline{x} \times \underline{\nabla}) A^{i}(x)\right]\right| P, S\right\rangle .
$$

This agrees with the first term in the second line of eq. (6.39) in [7]. Note that $\underline{\nabla}^{i}=$ $\partial / \partial x^{i}=\partial_{i}=-\partial^{i}$.

The OAM definitions above in eq. (2.1) (applied to gluons) and in eq. (29) of [18] (labeled HNXYZ) are very similar, and in fact would be identical if

$$
\int d b^{-} P^{+} \frac{1}{(2 \pi)^{3}} W^{\text {here }}(p, b)=W^{\mathrm{HNXYZ}}(x, \underline{p}, \underline{b}) .
$$

Using the Wigner distributions from eq. (25) in [18] and eq. (3.3) above we see that eq. (C.5) is satisfied if

$$
\begin{gathered}
\int \frac{d^{2} \Delta}{(2 \pi)^{2}}\left\langle P+\frac{\Delta}{2}, S\left|\operatorname{tr}\left[F^{+i}\left(\underline{b}+\frac{z}{2}\right) \mathcal{U} F^{+i}\left(\underline{b}-\frac{z}{2}\right)\right]\right| P-\frac{\Delta}{2}, S\right\rangle \\
=2 P^{+} \int d b^{-}\left\langle\operatorname{tr}\left[F^{+i}\left(b+\frac{z}{2}\right) \mathcal{U} F^{+i}\left(b-\frac{z}{2}\right)\right]\right\rangle,
\end{gathered}
$$

where we have replaced $\xi \rightarrow-z$ in eq. (3.3). The gauge link or links are denoted by a single $\mathcal{U}$ for brevity. Using eq. (A.4) we see that eq. (C.6) is indeed correct since

$$
\begin{aligned}
2 P^{+} & \int d b^{-}\left\langle\operatorname{tr}\left[F^{+i}\left(b+\frac{z}{2}\right) \mathcal{U} F^{+i}\left(b-\frac{z}{2}\right)\right]\right\rangle \\
& =2 P^{+} \int d b^{-} \frac{1}{2 P^{+}} \int \frac{d^{2} \Delta d \Delta^{+}}{(2 \pi)^{3}} e^{i b \cdot \Delta}\left\langle P+\frac{\Delta}{2}, S\left|\operatorname{tr}\left[F^{+i}\left(\frac{z}{2}\right) \mathcal{U} F^{+i}\left(-\frac{z}{2}\right)\right]\right| P-\frac{\Delta}{2}, S\right\rangle \\
& =\int \frac{d^{2} \Delta}{(2 \pi)^{2}} e^{-i \underline{b} \cdot \Delta}\left\langle P+\frac{\Delta}{2}, S\left|\operatorname{tr}\left[F^{+i}\left(\frac{z}{2}\right) \mathcal{U} F^{+i}\left(-\frac{z}{2}\right)\right]\right| P-\frac{\Delta}{2}, S\right\rangle \\
& =\int \frac{d^{2} \Delta}{(2 \pi)^{2}}\left\langle P+\frac{\Delta}{2}, S\left|\operatorname{tr}\left[F^{+i}\left(\underline{b}+\frac{z}{2}\right) \mathcal{U} F^{+i}\left(\underline{b}-\frac{z}{2}\right)\right]\right| P-\frac{\Delta}{2}, S\right\rangle .
\end{aligned}
$$

Hence our gluon OAM definition is equivalent to that in $[7,18]$.

\section{Large nucleus limit}

Imagine the CGC limit, that is, consider the proton to be a large nucleus in the McLerranVenugopalan (MV) model [55-57] with one of the quarks in one of the nucleons polarized. 
In this case, at Born level, we can write using eq. (3.58)

$$
G_{10}^{i}=-\frac{\alpha_{s}^{2} C_{F}}{N_{c}} \pi \epsilon^{i j} x_{10}^{j} \ln \frac{1}{x_{10} \Lambda} T(b),
$$

where $T(b)$ is the nuclear profile function and, as usual, $\underline{b}=\left(\underline{x}_{0}+\underline{x}_{1}\right) / 2$. (See section 4.2.1 of [49], in particular eq. (4.32).)

Inserting eq. (D.1) into eq. (3.19) and observing that

$$
\int d^{2} b b^{j} T(b)=0
$$

due to the rotational symmetry of $T(b)$ we see that the first term in the right of eq. (3.19) vanishes and one arrives at

$$
\frac{d L_{G}}{d x}=-\frac{1}{2} \Delta G\left(x, Q^{2}\right)
$$

Below eq. (3.22) we made a comment that for the proton target modeled to be a single quark the relation between the gluon helicity and OAM (at the same Born level) is

$$
L_{G}\left(x, Q^{2}\right)=-\Delta G\left(x, Q^{2}\right),
$$

which is also in agreement with the parton model argument presented in appendix B of [18]. It appears that in the same Born (two-gluon exchange) approximation, the relation between the gluon helicity and OAM is different for the large-nucleus and single-quark targets, given by eqs. (D.3) and (D.4) respectively.

Interestingly, if we assume that (note the argument of $T$ )

$$
G_{10}^{i}=-\frac{\alpha_{s}^{2} C_{F}}{N_{c}} \pi \epsilon^{i j} x_{10}^{j} \ln \frac{1}{x_{10} \Lambda} T\left(x_{1}\right),
$$

or, in general that $G_{10}^{i} \sim T\left(x_{1}\right)$, then, employing eq. (3.19) again (or eq. (3.24)) we get

$$
L_{G}\left(x, Q^{2}\right)=0 .
$$

It seems that slightly different assumptions about the argument of $T$, equivalent under the large-nucleus approximation of the MV model, give significantly different results for $L_{G}$.

Indeed, we may vary the transverse position in the argument of the nuclear profile function by writing

$$
G_{10}^{i}=-\frac{\alpha_{s}^{2} C_{F}}{N_{c}} \pi \epsilon^{i j} x_{10}^{j} \ln \frac{1}{x_{10} \Lambda} T\left[\xi \underline{x}_{1}+(1-\xi) \underline{x}_{0}\right]
$$

with $\xi$ a real dimensionless number. Then repeating the above steps used in arriving at eq. (3.24) and eq. (D.6) we get

$$
L_{G}\left(x, Q^{2}\right)=(\xi-1) \Delta G\left(x, Q^{2}\right) .
$$


Our result (3.73) above corresponds to

$$
\xi=1+\frac{\alpha_{h}^{q}}{4} \ln \frac{Q^{2}}{\Lambda^{2}},
$$

that is, to $\xi$ slightly larger than 1 . Note that $\xi \approx 1$ gives $T\left(\underline{x}_{1}\right)$ in eq. (D.7), indicating that the position of the polarized (anti)quark is "more important". This is consistent with other findings in this work.

In the framework of the simple toy model for the polarized amplitude in eq. (D.7), our conclusion (3.73) in the main text appears to imply that the gluon OAM begins with $\xi=0$ in the initial conditions (at the Born level) corresponding to $L_{G}=-\Delta G$, and then, via the DLA evolution, this parameter $\xi$ evolves to $\xi \gtrsim 1$, as given by eq. (D.9), with the relation (3.73) between the gluon OAM and helicity. The physical reason between such a "center-of-mass" shift is not clear at present.

Open Access. This article is distributed under the terms of the Creative Commons Attribution License (CC-BY 4.0), which permits any use, distribution and reproduction in any medium, provided the original author(s) and source are credited.

\section{References}

[1] Y.V. Kovchegov, D. Pitonyak and M.D. Sievert, Helicity evolution at small x, JHEP 01 (2016) 072 [Erratum JHEP 10 (2016) 148] [arXiv: 1511.06737] [INSPIRE].

[2] Y.V. Kovchegov, D. Pitonyak and M.D. Sievert, Small-x asymptotics of the quark helicity distribution, Phys. Rev. Lett. 118 (2017) 052001 [arXiv:1610.06188] [INSPIRE].

[3] Y.V. Kovchegov, D. Pitonyak and M.D. Sievert, Helicity Evolution at Small x: Flavor Singlet and Non-Singlet Observables, Phys. Rev. D 95 (2017) 014033 [arXiv:1610.06197] [INSPIRE].

[4] Y.V. Kovchegov, D. Pitonyak and M.D. Sievert, Small-x Asymptotics of the Quark Helicity Distribution: Analytic Results, Phys. Lett. B 772 (2017) 136 [arXiv:1703. 05809] [INSPIRE].

[5] Y.V. Kovchegov, D. Pitonyak and M.D. Sievert, Small-x Asymptotics of the Gluon Helicity Distribution, JHEP 10 (2017) 198 [arXiv:1706.04236] [INSPIRE].

[6] Y.V. Kovchegov and M.D. Sievert, Small-x Helicity Evolution: an Operator Treatment, arXiv: 1808.09010 [INSPIRE].

[7] R.L. Jaffe and A. Manohar, The $g_{1}$ Problem: Fact and Fantasy on the Spin of the Proton, Nucl. Phys. B 337 (1990) 509 [InSPIRE].

[8] X.-d. Ji, Gauge-Invariant Decomposition of Nucleon Spin, Phys. Rev. Lett. 78 (1997) 610 [hep-ph/9603249] [INSPIRE].

[9] X.-d. Ji, X. Xiong and F. Yuan, Proton Spin Structure from Measurable Parton Distributions, Phys. Rev. Lett. 109 (2012) 152005 [arXiv:1202.2843] [INSPIRE].

[10] E. Leader and C. Lorcé, The angular momentum controversy: What's it all about and does it matter?, Phys. Rept. 541 (2014) 163 [arXiv:1309.4235] [INSPIRE].

[11] S. Bashinsky and R.L. Jaffe, Quark and gluon orbital angular momentum and spin in hard processes, Nucl. Phys. B 536 (1998) 303 [hep-ph/9804397] [INSPIRE]. 
[12] P. Hagler and A. Schafer, Evolution equations for higher moments of angular momentum distributions, Phys. Lett. B 430 (1998) 179 [hep-ph/9802362] [INSPIRE].

[13] A. Harindranath and R. Kundu, On Orbital angular momentum in deep inelastic scattering, Phys. Rev. D 59 (1999) 116013 [hep-ph/9802406] [InSPIRE].

[14] Y. Hatta and S. Yoshida, Twist analysis of the nucleon spin in QCD, JHEP 10 (2012) 080 [arXiv: 1207.5332] [INSPIRE].

[15] X.-d. Ji, X. Xiong and F. Yuan, Probing Parton Orbital Angular Momentum in Longitudinally Polarized Nucleon, Phys. Rev. D 88 (2013) 014041 [arXiv:1207.5221] [INSPIRE].

[16] Y. Hatta and D.-J. Yang, On the small-x behavior of the orbital angular momentum distributions in QCD, Phys. Lett. B 781 (2018) 213 [arXiv:1802.02716] [INSPIRE].

[17] O. Martin, P. Hagler and A. Schafer, Numerical solution of the evolution equation for orbital angular momentum of partons in the nucleon, Phys. Lett. B 448 (1999) 99 [hep-ph/9810474] [INSPIRE].

[18] Y. Hatta, Y. Nakagawa, F. Yuan, Y. Zhao and B. Xiao, Gluon orbital angular momentum at small-x, Phys. Rev. D 95 (2017) 114032 [arXiv: 1612.02445] [INSPIRE].

[19] E.A. Kuraev, L.N. Lipatov and V.S. Fadin, The Pomeranchuk Singularity in Nonabelian Gauge Theories, Sov. Phys. JETP 45 (1977) 199 [InSPIRE].

[20] I.I. Balitsky and L.N. Lipatov, The Pomeranchuk Singularity in Quantum Chromodynamics, Sov. J. Nucl. Phys. 28 (1978) 822 [inSPIRE].

[21] I.I. Balitsky, Operator expansion for high-energy scattering, Nucl. Phys. B 463 (1996) 99 [hep-ph/9509348] [INSPIRE].

[22] I.I. Balitsky, Factorization and high-energy effective action, Phys. Rev. D 60 (1999) 014020 [hep-ph/9812311] [INSPIRE].

[23] Y.V. Kovchegov, Small-x $F_{2}$ structure function of a nucleus including multiple Pomeron exchanges, Phys. Rev. D 60 (1999) 034008 [hep-ph/9901281] [INSPIRE].

[24] Y.V. Kovchegov, Unitarization of the BFKL Pomeron on a nucleus, Phys. Rev. D 61 (2000) 074018 [hep-ph/9905214] [InSPIRE].

[25] J. Jalilian-Marian, A. Kovner and H. Weigert, The Wilson renormalization group for low $x$ physics: Gluon evolution at finite parton density, Phys. Rev. D 59 (1998) 014015 [hep-ph/9709432] [INSPIRE].

[26] J. Jalilian-Marian, A. Kovner, A. Leonidov and H. Weigert, The Wilson renormalization group for low x physics: Towards the high density regime, Phys. Rev. D 59 (1998) 014014 [hep-ph/9706377] [INSPIRE].

[27] E. Iancu, A. Leonidov and L.D. McLerran, The Renormalization group equation for the color glass condensate, Phys. Lett. B 510 (2001) 133 [hep-ph/0102009] [INSPIRE].

[28] E. Iancu, A. Leonidov and L.D. McLerran, Nonlinear gluon evolution in the color glass condensate. 1., Nucl. Phys. A 692 (2001) 583 [hep-ph/0011241] [InSPIRE].

[29] R. Kirschner and L.N. Lipatov, Double Logarithmic Asymptotics and Regge Singularities of Quark Amplitudes with Flavor Exchange, Nucl. Phys. B 213 (1983) 122 [INSPIRE].

[30] R. Kirschner, Regge Asymptotics of Scattering Amplitudes in the Logarithmic Approximation of QCD, Z. Phys. C 31 (1986) 135 [INSPIRE]. 
[31] R. Kirschner, Regge asymptotics of scattering with flavor exchange in QCD, Z. Phys. C 67 (1995) 459 [hep-th/9404158] [INSPIRE].

[32] R. Kirschner, Reggeon interactions in perturbative QCD, Z. Phys. C 65 (1995) 505 [hep-th/9407085] [INSPIRE].

[33] S. Griffiths and D.A. Ross, Studying the perturbative Reggeon, Eur. Phys. J. C 12 (2000) 277 [hep-ph/9906550] [InSPIRE].

[34] K. Itakura, Y.V. Kovchegov, L. McLerran and D. Teaney, Baryon stopping and valence quark distribution at small x, Nucl. Phys. A 730 (2004) 160 [hep-ph/0305332] [INSPIRE].

[35] J. Bartels and M. Lublinsky, Quark anti-quark exchange in $\gamma^{*} \gamma^{*}$ scattering, JHEP 09 (2003) 076 [hep-ph/0308181] [INSPIRE].

[36] J. Bartels, B.I. Ermolaev and M.G. Ryskin, Nonsinglet contributions to the structure function $g_{1}$ at small-x, Z. Phys. C 70 (1996) 273 [hep-ph/9507271] [INSPIRE].

[37] J. Bartels, B.I. Ermolaev and M.G. Ryskin, Flavor singlet contribution to the structure function $g_{1}$ at small-x, Z. Phys. C 72 (1996) 627 [hep-ph/9603204] [INSPIRE].

[38] A.V. Belitsky, X.-d. Ji and F. Yuan, Quark imaging in the proton via quantum phase space distributions, Phys. Rev. D 69 (2004) 074014 [hep-ph/0307383] [INSPIRE].

[39] C. Lorcé and B. Pasquini, Quark Wigner Distributions and Orbital Angular Momentum, Phys. Rev. D 84 (2011) 014015 [arXiv:1106.0139] [InSPIRE].

[40] Y. Hatta, Notes on the orbital angular momentum of quarks in the nucleon, Phys. Lett. B 708 (2012) 186 [arXiv:1111.3547] [INSPIRE].

[41] C. Lorcé, B. Pasquini, X. Xiong and F. Yuan, The quark orbital angular momentum from Wigner distributions and light-cone wave functions, Phys. Rev. D 85 (2012) 114006 [arXiv:1111.4827] [INSPIRE].

[42] P.J. Mulders and R.D. Tangerman, The complete tree-level result up to order $1 / Q$ for polarized deep-inelastic leptoproduction, Nucl. Phys. B 461 (1996) 197 [Erratum ibid. B 484 (1997) 538] [hep-ph/9510301] [INSPIRE].

[43] L.V. Gribov, E.M. Levin and M.G. Ryskin, Semihard Processes in QCD, Phys. Rept. 100 (1983) 1 [INSPIRE].

[44] E. Iancu and R. Venugopalan, The Color glass condensate and high-energy scattering in QCD, in Quark-Gluon Plasma 3, R.C. Hwa and X.-N. Wang eds., World Scientific (2004), pp. 249-363 [hep-ph/0303204] [INSPIRE].

[45] H. Weigert, Evolution at small $x_{\mathrm{bj}}$ : The Color glass condensate, Prog. Part. Nucl. Phys. 55 (2005) 461 [hep-ph/0501087] [InSPIRE].

[46] J. Jalilian-Marian and Y.V. Kovchegov, Saturation physics and deuteron-Gold collisions at RHIC, Prog. Part. Nucl. Phys. 56 (2006) 104 [hep-ph/0505052] [INSPIRE].

[47] F. Gelis, E. Iancu, J. Jalilian-Marian and R. Venugopalan, The Color Glass Condensate, Ann. Rev. Nucl. Part. Sci. 60 (2010) 463 [arXiv:1002.0333] [inSPIRE].

[48] J.L. Albacete and C. Marquet, Gluon saturation and initial conditions for relativistic heavy ion collisions, Prog. Part. Nucl. Phys. 76 (2014) 1 [arXiv:1401.4866] [INSPIRE].

[49] Y.V. Kovchegov and E. Levin, Quantum Chromodynamics at High Energy, Cambridge University Press (2012). 
[50] G.P. Lepage and S.J. Brodsky, Exclusive Processes in Perturbative Quantum Chromodynamics, Phys. Rev. D 22 (1980) 2157 [InSPIRE].

[51] Y.V. Kovchegov and M.D. Sievert, Valence Quark Transversity at Small x, arXiv:1808.10354 [INSPIRE].

[52] A.H. Mueller and S. Munier, $p_{\perp}$-broadening and production processes versus dipole/quadrupole amplitudes at next-to-leading order, Nucl. Phys. A 893 (2012) 43 [arXiv: 1206.1333] [INSPIRE].

[53] F. Dominguez, C. Marquet, B.-W. Xiao and F. Yuan, Universality of Unintegrated Gluon Distributions at small x, Phys. Rev. D 83 (2011) 105005 [arXiv:1101.0715] [INSPIRE].

[54] G.A. Chirilli, Y.V. Kovchegov and D.E. Wertepny, Regularization of the Light-Cone Gauge Gluon Propagator Singularities Using Sub-Gauge Conditions, JHEP 12 (2015) 138 [arXiv: 1508.07962] [INSPIRE].

[55] L.D. McLerran and R. Venugopalan, Computing quark and gluon distribution functions for very large nuclei, Phys. Rev. D 49 (1994) 2233 [hep-ph/9309289] [InSPIRE].

[56] L.D. McLerran and R. Venugopalan, Gluon distribution functions for very large nuclei at small transverse momentum, Phys. Rev. D 49 (1994) 3352 [hep-ph/9311205] [INSPIRE].

[57] L.D. McLerran and R. Venugopalan, Green's functions in the color field of a large nucleus, Phys. Rev. D 50 (1994) 2225 [hep-ph/9402335] [INSPIRE]. 